\title{
IL-15 triggers an antiapoptotic pathway in human intraepithelial lymphocytes that is a potential new target in celiac disease-associated inflammation and lymphomagenesis
}

\author{
Georgia Malamut,1,2,3 Raja El Machhour,1,2 Nicolas Montcuquet,1,2 \\ Séverine Martin-Lannerée, ${ }^{4,5}$ Isabelle Dusanter-Fourt, 4,5 Virginie Verkarre, ,,2,6 \\ Jean-Jacques Mention, ${ }^{1,2}$ Gabriel Rahmi, ${ }^{3}$ Hiroshi Kiyono, ${ }^{7}$ Eric A. Butz, ${ }^{8}$ Nicole Brousse, ,1,2,6 \\ Christophe Cellier, ${ }^{1,3}$ Nadine Cerf-Bensussan, ${ }^{1,2}$ and Bertrand Meresse ${ }^{1,2}$
}

${ }^{1}$ INSERM U989, Paris, France. ${ }^{2}$ Université Paris Descartes, Faculté de Médecine René Descartes, Paris, France. ${ }^{3}$ Department of Gastroenterology, AP-HP, Hôpital Européen Georges Pompidou, Paris, France. ${ }^{4}$ Institut Cochin, Université Paris Descartes, CNRS (UMR 8104), Paris, France. ${ }^{5}$ INSERM U1016, Paris, France. ${ }^{6}$ Department of Pathology, AP-HP, Hôpital Necker-Enfants Malades, Paris, France. ${ }^{7}$ Division of Mucosal Immunology, Department of Microbiology and Immunology, Institute of Medical Science, University of Tokyo, Tokyo, Japan. ${ }^{8}$ Inflammation Department, AMGEN Inc., Seattle, Washington, USA.

\begin{abstract}
Enteropathy-associated $\mathrm{T}$ cell lymphoma is a severe complication of celiac disease (CD). One mechanism suggested to underlie its development is chronic exposure of intraepithelial lymphocytes (IELs) to potent antiapoptotic signals initiated by IL-15, a cytokine overexpressed in the enterocytes of individuals with CD. However, the signaling pathway by which IL-15 transmits these antiapoptotic signals has not been firmly established. Here we show that the survival signals delivered by IL-15 to freshly isolated human IELs and to human IEL cell lines derived from CD patients with type II refractory CD (RCDII) - a clinicopathological entity considered an intermediary step between $C D$ and enteropathy-associated $T$ cell lymphoma - depend on the antiapoptotic factors Bcl-2 and/or Bcl-xL. The signals also required IL-15R $\beta$, Jak3, and STAT5, but were independent of PI3K, ERK, and STAT3. Consistent with these data, IELs from patients with active CD and RCDII contained increased amounts of Bcl-xL, phospho-Jak3, and phospho-STAT5. Furthermore, incubation of patient duodenal biopsies with a fully humanized human IL-15-specific Ab effectively blocked Jak3 and STAT5 phosphorylation. In addition, treatment with this Ab induced IEL apoptosis and wiped out the massive IEL accumulation in mice overexpressing human IL-15 in their gut epithelium. Together, our results delineate the IL-15-driven survival pathway in human IELs and demonstrate that IL-15 and its downstream effectors are meaningful therapeutic targets in RCDII.
\end{abstract}

\section{Introduction}

Celiac disease (CD) is a model disease to analyze how the loss of oral tolerance to dietary proteins, the cereal-derived prolamines (collectively called gluten), can lead to chronic intestinal inflammation, systemic autoimmunity, and ultimately $\mathrm{T}$ cell lymphomagenesis (1). Indeed, one severe complication of CD can be the onset of enteropathy-associated T cell lymphoma (EATL), a high-grade invasive lymphoma with a very poor prognosis that occurs late in adult life, usually after years of undiagnosed and/or silent CD (2). Our previous work has demonstrated that EATL arises from intraepithelial lymphocytes (IELs; refs. 3, 4). This unusual T cell population undergoes a massive expansion in $\mathrm{CD}$ and participates in a cytolytic attack of the epithelium orchestrated by IL-15, a potent proinflammatory cytokine upregulated in the intestinal epithelium of CD patients $(5,6)$. In CD, this cytokine stimulates the effector functions of IELs and their cytotoxicity against epithelial cells,

Authorship note: Georgia Malamut and Raja El Machhour contributed equally to this work.

Conflict of interest: The authors have declared that no conflict of interest exists. Citation for this article: J Clin Invest. 2010;120(6):2131-2143. doi:10.1172/JCI41344. notably by increasing their expression of activating NK receptors and/or synergizing with the NK receptor signaling cascade $(7,8)$. IL-15 might also be instrumental in driving lymphomagenesis within the IEL compartment. This hypothesis is consistent with the observation that IL-15 transgenic mice develop CD8/NK lymphomas and leukemias (9). In these mice, chronic overexpression of IL-15 may pervert the normal homeostatic prosurvival signals delivered by IL- 15 to $\mathrm{NK}$ and $\mathrm{CD}^{+} \mathrm{T}$ lymphocytes into an abnormal protracted antiapoptotic signal impairing their elimination after activation or upon transformation, thereby allowing their accumulation and additional transformation events (9). Along this line, we and others have obtained evidence that IL-15 contributes to enhanced survival and subsequent accumulation of nontransformed IELs in CD (10) as well as of aberrant IELs in type II refractory CD (RCDII; ref. 6). The mechanisms that drive IL- 15 secretion in CD remain to be determined. We and others have provided evidence that exposure to gluten peptides can upregulate IL-15 synthesis in intestinal tissues of $\operatorname{CD}(5,6,11)$, yet the nature of the posttranslational mechanism underlying IL-15 upregulation in CD remains unknown. Notably, it is unclear whether gluten acts directly or indirectly by promoting tissue inflammation and/or 
stress. This second possibility is supported by the observation that in RCDII, overexpression of IL-15 persists or resumes together with villous atrophy despite the gluten-free diet (GFD; ref. 6). RCDII is a new clinicopathological entity now considered as an intraepithelial $\mathrm{T}$ cell lymphoma and has recently been identified as an intermediary step between CD and EATL. RCDII is characterized by phenotypically aberrant IELs, which lack surface CD3$\mathrm{T}$ cell receptor complexes ( $\mathrm{sCD}^{-}$) but contain several CD3 chains (refs. 3, 12, and our unpublished observations). Abnormal IELs generally lack CD8, but, like their normal counterparts, express CD45, CD7, and the CD103 integrin, a marker shared by EATL $(4,13)$. In contrast to EATL cells, RCDII IELs display normal cytological features and do not proliferate in situ $(6,13)$. However, they contain clonal TCR $\gamma$ gene rearrangements $(3,13)$ and chromosomal abnormalities (14) and progressively replace and/or eliminate the normal subsets of $\mathrm{CD}^{+} \mathrm{CD}^{+}{ }^{+} \mathrm{TCR} \alpha \beta^{+}$and $\mathrm{CD} 8^{+/-} \mathrm{CD} 3^{+} \mathrm{TCR} \gamma \delta^{+}$ IELs. Furthermore, they can disseminate into the lamina propria, blood, and extraintestinal sites (15), all features strongly suggestive of their malignant nature. Moreover, approximately $40 \%$ of RCDII patients develop EATL, which, when tested, contains the same clonal TCR $\gamma$ rearrangements as do RCDII IELs (15), verifying the intraepithelial origin of EATL and attesting that RCDII is as an intermediate stage in the process leading from hyperplasia of normal $\mathrm{T}$ IELs in CD to overt $\mathrm{T}$ cell lymphomas.

Eradicating abnormal IELs at the stage of RCDII should prevent the onset of EATL. However, this goal has not been attainable by various immunosuppressive and chemotherapeutic approaches, and the prognosis of RCDII remains very poor (15). IL-15-dependent antiapoptotic signaling is a possible therapeutic target. Whereas the potent antiapoptotic properties of IL-15 in $\mathrm{CD}^{+} \mathrm{T}$ cells and NK cells are definitively established $(16,17)$, the signaling pathway is not firmly established. Recent studies in murine NK cells have emphasized the role of the PI3K pathway and of Mcl-1 (18), yet it is unclear whether this pathway can be generalized to human T cells, either normal or transformed. Here, we show that IL-15 elicits the same antiapoptotic signaling cascade implicating Jak3, STAT5, and Bcl-xL in normal and RCDII IEL cell lines and demonstrate its in situ activation in active CD (ACD) and RCDII, thus providing a strong rationale for a strategy based on IL-15 blockade to treat RCDII.

\section{Results}

IL-15-induced survival of RCDII IEL lines depends on Bcl-2 and/or $B c l-x L$, but not $M c l-1$. One important mechanism underlying the potent prosurvival signal delivered by IL-15 might be the induction of antiapoptotic factors of the Bcl-2 family. Thus, the antiapoptotic effect of IL-15 in NK cells was ascribed to the induction of either Bcl-2 in humans (19) or Mcl-1 in mice (18), whereas the contribution of a third prosurvival factor induced by IL-15, Bcl-xL, was suggested in human NK cells (20), murine memory $\mathrm{T}$ lymphocytes (21), and intestinal IELs (22). We therefore examined the role of these factors in IL-15-driven survival of RCDII IEL lines. All 3 factors - Bcl-2, Bcl-xL, and Mcl-1 - were detected by Western blot, and Bcl-2 and Bcl-xL were detected by intracellular staining. In RCDII IEL lines, this expression remained detectable at $0.5 \mathrm{ng} / \mathrm{ml} \mathrm{IL-15}$, the lowest concentration able to maintain lymphocyte survival (Figure 1, A and B). IL-15 starvation resulted in strong apoptosis of RCDII IEL lines accompanied by a progressive increase in the percentage of cells stained by annexin $\mathrm{V}$ and propidium iodide (PI) between 48 and 96 hours. In keeping with a role for the intrinsic pathway, cleavage of caspases 9 and 3 was also observed (Supplemental Figure 1; supplemental material available online with this article; doi:10.1172/JCI41344DS1). A rapid and profound decrease of $\mathrm{Bcl}-\mathrm{xL}$ and $\mathrm{Bcl}-2$ transcription was visible at 6 hours (Figure 1C) and persisted after 48 hours. In contrast, $M c l-1$ mRNA decreased only modestly at early time points, and even tended to increase after 48 hours of IL-15 deprivation (Figure $1 \mathrm{C}$ ). Decreased intracellular expression of Bcl-2 and Bcl-xL was detected by flow cytometry analysis by 48 hours and was more profound for Bcl-xL than for Bcl-2 (Figure 1B). Western blot analysis at 72 hours showed decreased levels of the 3 antiapoptotic molecules, but statistical analysis of 5 distinct RCDII IEL lines indicated that the decrease was only significant for $\mathrm{Bcl}-\mathrm{xL}$ $(P=0.04$, paired $t$ test; Figure $1 \mathrm{~A})$, suggestive of a preferential influence of IL-15 on the regulation of this antiapoptotic factor in the human IEL lines studied.

Prosurvival factors of the Bcl-2 family are made of 3 domains, $\mathrm{BH} 1, \mathrm{BH} 2$, and $\mathrm{BH} 3$, which fold together to form a receptor site that binds proapoptotic $\mathrm{BH} 3$-only proteins, thereby protecting cells from apoptosis induction (23). The $\mathrm{BH} 3$ mimetic ABT-737 binds $\mathrm{Bcl}-2$ and $\mathrm{Bcl}-\mathrm{xL}$, preventing their association with proapoptotic molecules and neutralizing their antiapoptotic activity, but not the activity of Mcl-1 (24). A 48-hour treatment of IL-15dependent RCDII IEL lines with increasing concentrations of ABT-737 resulted in a dramatic induction of apoptosis and a marked decrease in the number of live lymphocytes (Figure 1D). Not surprisingly, ABT-737, which directly blocks Bcl-2 and Bcl-xL function, induced apoptosis more rapidly than did IL-15 starvation, which markedly reduced the intracellular levels of the 2 proteins after 48 hours. Together, these findings demonstrate that in RCDII IEL lines, Bcl-xL and/or Bcl-2 are central to the prosurvival effect of IL-15, but Mcl-1 is not.

IL-15 promotes survival of abnormal IELs through stimulation of the IL-15 $\beta \gamma$ receptor and activation by the Jak3/STAT5 signaling pathway. The IL-15 receptor is composed of a specific but widely expressed $\alpha$ chain (IL-15R $\alpha$ ) and the $\beta$ and $\gamma_{c}$ chains, which are shared with the IL-2 receptor and coexpressed exclusively by lymphocytes (IL-2/ IL-15R $\beta \gamma_{c}$ ). The exact contribution of IL-15R $\alpha$ to IL-15 signaling is controversial. IL- $15 \mathrm{R} \alpha$ can confer high avidity and cytokine specificity to the receptor when expressed on lymphocytes (25). An autonomous role in the delivery of antiapoptotic signals was also suggested (16). However, a series of elegant studies provided evidence that NK cell and IEL development and homeostasis require IL-15R $\alpha$ only to chaperone IL-15 to the surface of a variety of hematopoietic and nonhematopoietic cells and permit its efficient trans-presentation to lymphocytes expressing IL-2/IL-15R $\beta \gamma_{c}$ $(26,27)$. A 72 -hour incubation of RCDII IEL lines in the presence of anti-IL-15R $\beta$ mAb completely blocked IL-15-mediated survival $(P=0.02$, paired $t$ test; Figure $2 \mathrm{~A})$ and strongly inhibited expression of Bcl-xL and, to a lesser degree, Bcl-2 (Figure 2B). In contrast, a blocking anti-IL-15R $\alpha \mathrm{mAb}$ had no effect, which indicates that the $\beta \gamma_{c}$ receptor alone is necessary and sufficient to transduce IL-15derived survival signals in the studied cell lines.

In normal $\mathrm{T}$ lymphocytes, the IL- $2 / \mathrm{IL}-15 \mathrm{R} \beta \gamma_{\mathrm{c}}$ receptor can activate several pathways that may control lymphocyte survival, including the AKT and ERK pathways and Jak-dependent activation of STAT3 and STAT5 $(17,28)$. AKT, ERK, Jak3, STAT3, and STAT5 were all phosphorylated in RCDII IEL lines, and their phosphorylation decreased markedly either after IL-15 starvation or in the presence of anti-IL-15R $\beta \mathrm{Ab}$, whereas anti-IL-15R $\alpha$ 
A

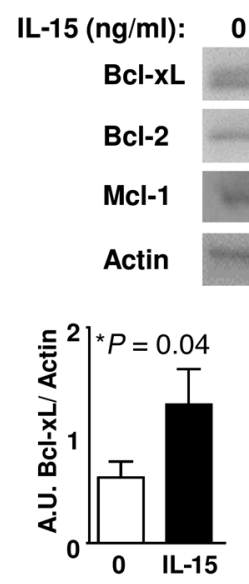

RCDII patient 1 RCDIl patient 2
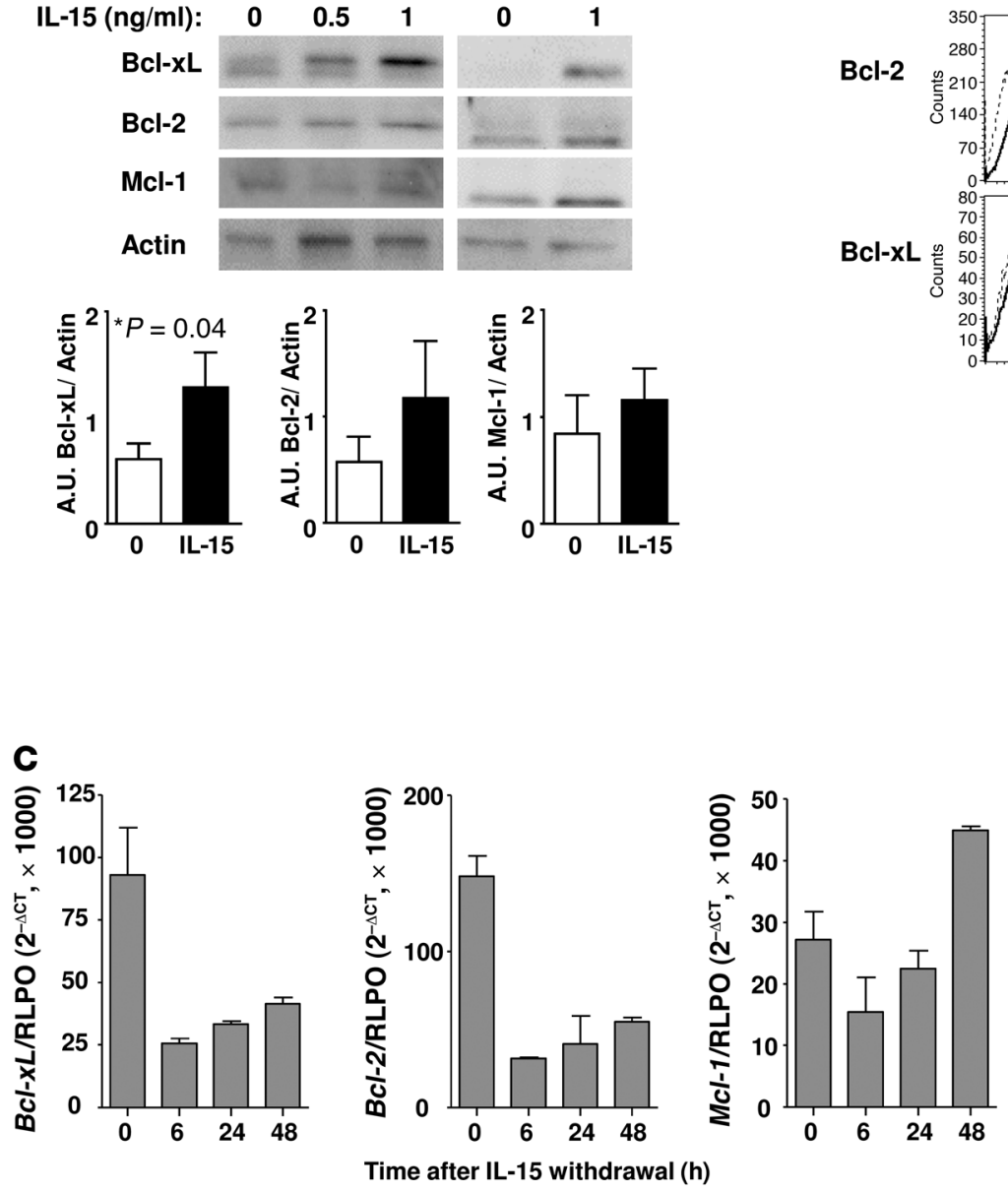

B IL-15 (ng/ml): 0

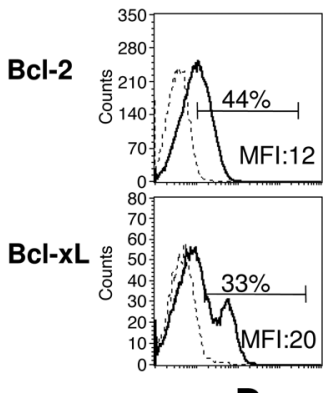

0.5
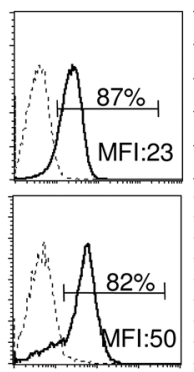

D

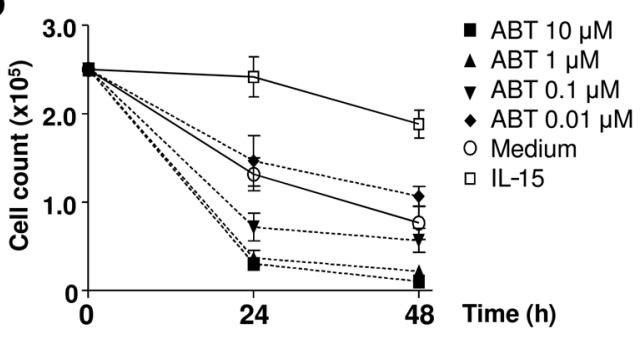

Figure 1

IL-15-induced survival of RCDII IEL lines depends on Bcl-2 and/or Bcl-xL. (A) Western blot analysis. Representative blots show Bcl-2, Bcl-xL, and Mcl-1 in 2 different RCDII IEL lines cultured for 72 hours with the indicated concentrations of IL-15. Below, comparison by densitometry of $\mathrm{Bcl}-2, \mathrm{Bcl}-\mathrm{xL}$, and $\mathrm{Mcl}-1$ levels in $4 \mathrm{RCDII} I \mathrm{EL}$ lines is shown normalized to $\beta$-actin. Only Bcl-xL expression significantly decreased upon IL-15 deprivation (paired $t$ test). (B) FACS analysis. Percentages of positive RCDII IELs and MFI of Bcl-2 and Bcl-xL decreased with IL-15 concentrations, but their expression remained detectable at $0.5 \mathrm{ng} / \mathrm{ml}$, the lowest concentration allowing cell survival. (C) mRNA levels for $B c l-2, B c l-x L$, and $\mathrm{Mcl}-1$. Only Bcl-2 and Bcl-xL mRNA levels decreased after IL-15 starvation in RCDII IEL lines. Shown is 1 representative experiment of 3. Values were normalized to ribosomal Protein Large PO (RLPO). (D) Apoptosis induction by ABT-737. RCDII IEL lines were cultured with $5 \mathrm{ng} / \mathrm{ml}$ IL-15 in the presence of ABT-737 at the indicated concentrations for 48 hours. Cell survival was assessed by counting viable cells in trypan blue (top) and by flow cytometry after staining with Annexin V/PI (bottom). Shown is 1 representative of 4 experiments with 4 RCDII IEL lines.

mAb had no effect (Figures 2, C and D). In order to delineate the pathways underlying the prosurvival effect of IL-15, RCDII IEL lines were treated with a panel of specific inhibitors. Strikingly, only the Jak3 inhibitor WHIP-131 significantly increased the percentage of apoptotic cells (untreated, $17 \% \pm 9 \%$; WHIP-131, 44\% $\pm 11 \% ; P<0.001$, 1-way ANOVA with Tukey's multiple comparison test; Figure 3A). Comparable results were obtained with a second inhibitor, PF-956980 (Supplemental Figure 4), recently reported to be highly specific for JAK3 (29). In contrast, neither the ERK inhibitor nor the PI3K inhibitors wortmannin and LY294002, which block signaling upstream of AKT, had a significant effect on IL-15-induced survival of RCDII IEL lines (Figure 3A). Accordingly, WHIP-131, but not LY294002, reduced Bcl-xL and Bcl-2 expression (Figure 3B). Notably, LY294002 and WHIP-131 strongly inhibited the proliferation of RCDII IEL lines triggered by a high $10-\mathrm{ng} / \mathrm{ml}$ IL-15 concentration (Figure 3C), demonstrating their efficacy and indicating that the role of the PI3K/AKT pathway is confined to the control of proliferation in the latter cells.

As shown in Figure 3D, WHIP-131 prevented the phosphorylation of both STAT3 and STAT5. To further confirm the role of the Jak3/STAT pathway and to delineate the respective contribution of STAT3 and STAT5, IL-15-dependent prosurvival signaling was next analyzed using shRNAs specifically targeting each of these transcription factors. shRNAs specific for STAT5 and STAT3 (shSTAT5 and shSTAT3, respectively) efficiently blocked the expression of their respective targets in transduced RCDII IEL lines (Figure 3E). Yet only STAT5 inhibition reduced expression of Bcl-xL and Bcl-2; increased the amount of cleaved caspase 3 (Figure 3E), a key downstream effector molecule in the apoptotic cascade; and simultaneously provoked a rapid decrease in cell viability (Figure 3F). Collectively, these results indicate that STAT5, but not STAT3, is necessary to prevent apopto- 
A

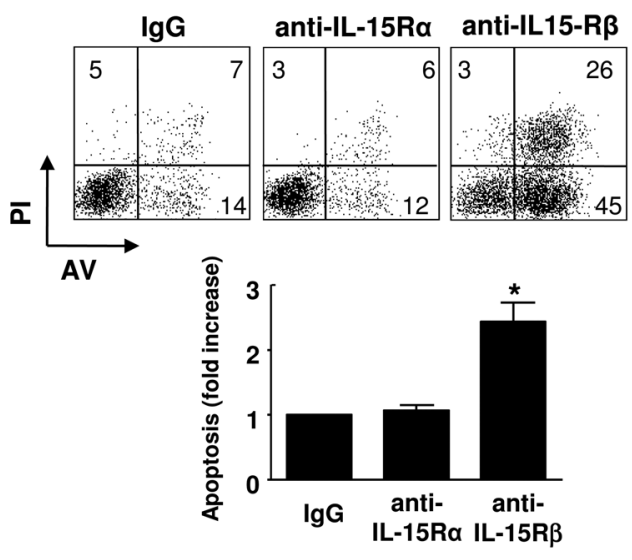

B

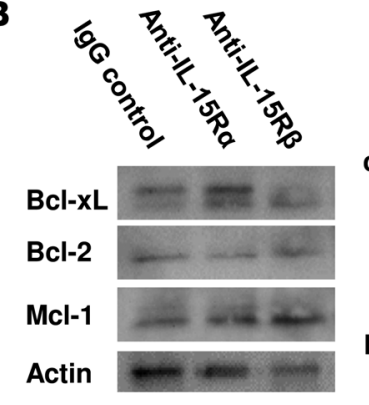

$\mathrm{Bcl}-2$

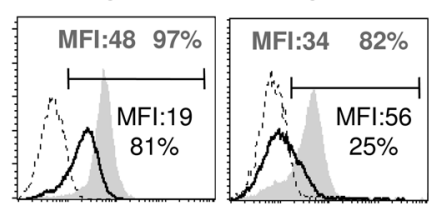

antiIL-15Ra
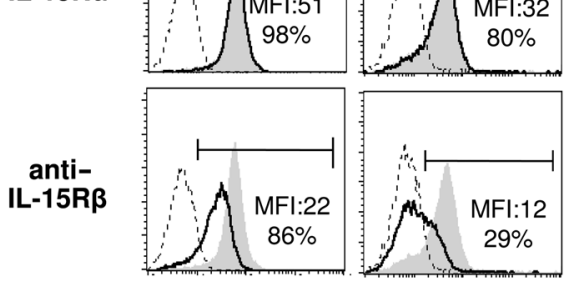

C IP: JAK3 Blot:

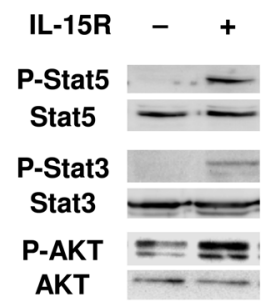

Histone 3

\section{JAK3}

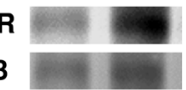

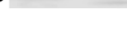

D

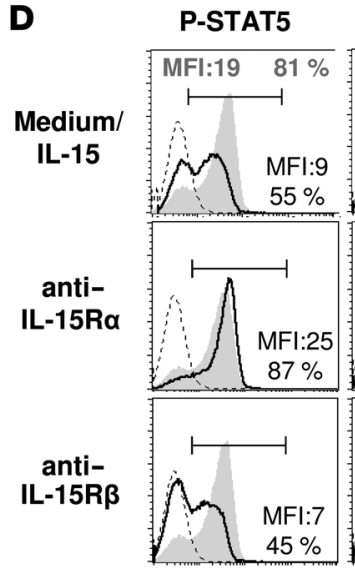

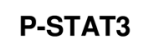

P-AKT
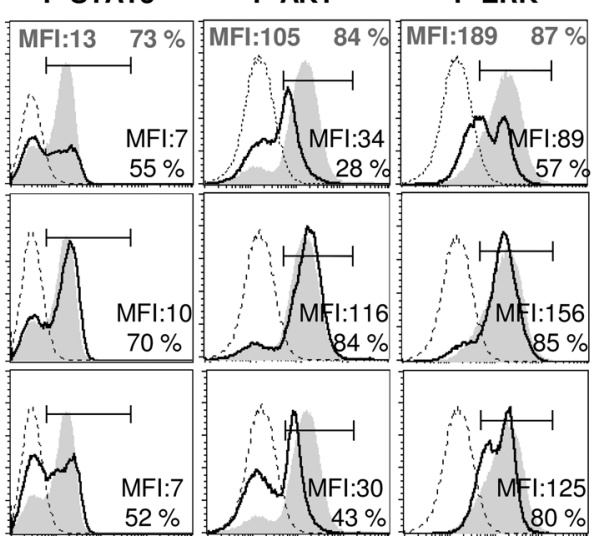

Figure 2

IL-15 survival signal depends on IL-15R $\beta \gamma_{c}$ in RCDII IEL lines. (A and B) Analysis of RCDII IEL lines cultured with $5 \mathrm{ng} / \mathrm{ml}$ IL-15 in the presence of anti-IL-15R $\alpha$ or anti-IL-15R $\beta$ blocking mAbs or control isotype for 72 hours. (A) Flow cytometry analysis of apoptosis in 1 representative cell line, and mean fold increase in percent apoptotic (annexin $\mathrm{V} / \mathrm{PI}+$ ) cells in $4 \mathrm{RCDII}$ IEL lines. Only anti-IL-15R $\beta$ mAb significantly increased apoptosis. ${ }^{*} P=0.02$ versus IgG, paired $t$ test. (B) Analysis of Bcl-2, Bcl-xL, and Mcl-1 expression by Western blot and of Bcl-2 and Bcl-xL expression (black histograms and text) by flow cytometry. Blockade of IL-15R $\beta$, but not IL-15R $\alpha$, decreased Bcl-xL, and to a lesser degree Bcl-2, compared with cells cultured in the presence of control isotype (gray filled histograms and text); Mcl-1 expression was unchanged. Shown is 1 representative experiment of 4. (C and D) Analysis of IL-15R $\beta$-dependent signaling in RCDII IEL lines. (C) Comparison of cell lines cultured with or without IL-15 for 72 hours demonstrated IL-15-induced phosphorylation of Jak3, STAT5, STAT3, and AKT. (D) Flow cytometry showed that IL-15 starvation and IL-15R $\beta$ blockade, but not IL-15R $\alpha$ blockade, decreased phosphorylation of STAT5, STAT3, AKT, and ERK (black histograms and text) compared with control cells in IL-15 (gray filled histograms and text). Dashed lines in A, B, and D denote isotype controls.

sis in RCDII IEL lines. Neither shSTAT5 nor shSTAT3 downmodulated Mcl-1 expression (Figure 3E), confirming that this factor does not contribute to IL-15 prosurvival signals in the tested lines.

IL-15 activates the same antiapoptotic signaling pathway in RCDII IELs and in normal IELs from ACD patients and controls. IL-15 is thought to stimulate the survival and subsequent accumulation not only of transformed IELs in RCDII (6), but also of normal IELs in uncomplicated CD (10). In RCDII, however, transformed IELs progressively replace the normal hyperplasic IELs, which suggests that transformation confers a survival advantage and raising the possibility that transformation modifies IL-15 signaling. As expected, IL-15 provided a strong antiapoptotic signal to normal IELs isolated from patients with ACD or from control individuals (Figure 4A and data not shown). This prosurvival signal was inhibited by ABT-737, even at the lowest concentrations of 0.1 and $0.01 \mu \mathrm{M}$ (Figure 4B), as well as by the anti-IL-15R $\beta$ Ab and WHIP-131, but not by the anti-
IL-15R $\alpha \mathrm{Ab}$ or by drugs blocking ERK or PI3K (Figure 4C). Accordingly, IL-15 upregulated Bcl-2 and Bcl-xL expression and STAT5 phosphorylation in normal IELs (Figure 5A). Collectively, these data indicate that IL-15 activates the same antiapoptotic cascade in normal and transformed human IELs. Yet at the same 5-ng/ml IL-15 concentration, normal IELs expressed less Bcl-xL and phospho-STAT5 than did RCDII IELs. Moreover, 5- to 10-ng/ml IL-15 concentrations were necessary to prevent apoptosis in normal IELs, higher than the concentration required for RCDII IELs (Figure 5C). This result suggested that the antiapoptotic pathway can be more readily elicited in RCDII IELs. Consistent with this hypothesis, the level of IL-15R $\beta$ expression was significantly higher in freshly isolated abnormal IELs than in residual normal IELs from the same RCDII patient $(15.8 \pm 3.6$ versus $9.1 \pm 3.2 ; P=0.025$, paired $t$ test; Figure $5 B$ ). Thus, low concentrations of IL-15 rescued RCDII IEL lines, but not normal IEL lines, from apoptosis (Figure 5C). 
A

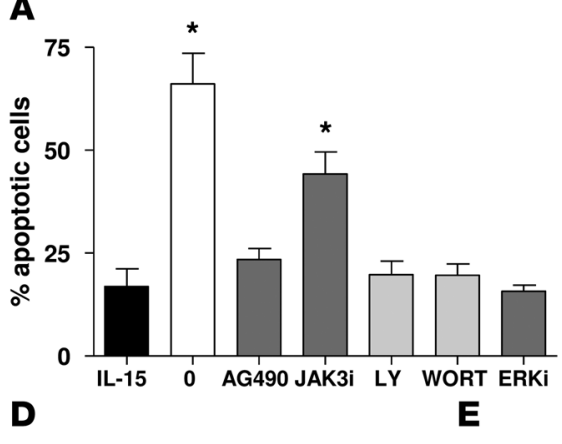

B

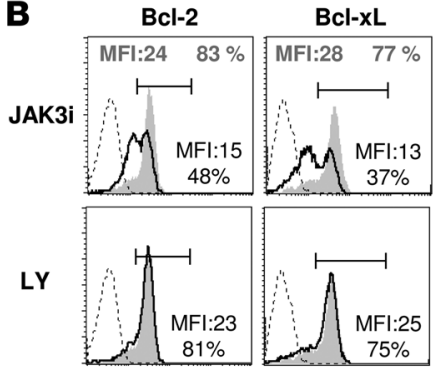

$\mathbf{F}$
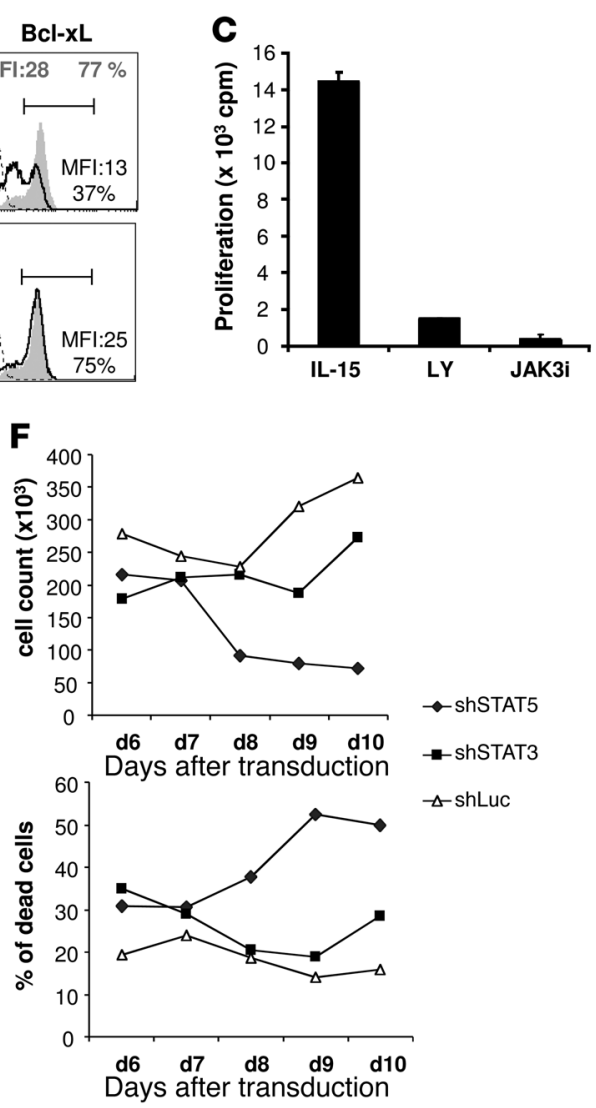

Figure 3

The Jak3/STAT5 signaling pathway is essential for IL-15-mediated survival of RCDII IEL lines. (A) Quantification of apoptosis in 5 RCDII IEL lines cultured for 72 hours with IL-15 in the presence of kinase inhibitors. Percentages of apoptotic cells, determined by flow cytometry as in Figure 2A, increased significantly after treatment with the Jak3 inhibitor WHIP-131 (JAK3i) and IL-15 starvation (0), but not with AG490, the PI3K inhibitors LY294002 and wortmannin, or the ERK inhibitor U0126 (ERKi). ${ }^{*} P<0.001$ versus IL-15 alone, 1-way ANOVA with Tukey's multiple comparison test. (B) Flow cytometry indicated that WHIP-131, but not LY294002 (black histograms and text), reduced expression of Bcl-2 and $\mathrm{Bcl}-\mathrm{xL}$ compared with control cells (gray filled histograms and text). (C) In contrast, proliferation of RCDII IEL lines to $10 \mathrm{ng} / \mathrm{ml}$ IL-15 assessed by ${ }_{3}^{3} \mathrm{H}$ thymidine incorporation was inhibited by both LY294002 and WHIP-131. Shown is 1 representative experiment of 3. (D) WHIP-131 decreased expression of phospho-STAT5 and phospho-STAT3 in treated cells (black histograms and text) compared with untreated cells (gray filled histograms and text). (E and F) Specific inhibition of STAT5 and STAT3 expression by shRNA in 1 RCDII IEL line cultured in IL-15. shLuc, control shRNA directed against the luciferase protein. (E) Analysis of signaling molecules and caspase 3 activation by Western blot. (F) Cell survival, measured as the number of viable cells and the percentage of trypan blue-positive (i.e., dead) cells. Inhibition of STAT5, but not STAT3, reduced expression of $\mathrm{Bcl}-2$ and $\mathrm{Bcl}-\mathrm{xL}$ and induced caspase 3 activation and cell death. Dashed lines in $\mathbf{B}$ and $\mathbf{D}$ denote isotype controls.

Increased expression of $B c l-x L$, but not Bcl-2, in the intestinal mucosa of patients with ACD and RCDII. Results obtained using IEL lines indicated that the antiapoptotic cascade operated by IL-15 via stimulation of IL-2/IL-15 $\beta \gamma_{c}$ involves successive activation of Jak3 and STAT5, which in turn drives expression of Bcl-xL and, although perhaps less efficiently, Bcl-2. To determine which antiapoptotic factor operates in CD and RCDII, expression of Bcl-xL and $\mathrm{Bcl}-2$ was compared in the intestinal mucosa of control individuals, of patients with uncomplicated CD (either ACD or on GFD), and of patients with RCDII. Western blot analysis of total duodenal extracts revealed a significant increase of $\mathrm{Bcl}-\mathrm{xL}$ in $\mathrm{ACD}$, GFD, and RCDII patients compared with controls (Figure 6A). Immunohistochemistry of duodenal biopsies revealed that $\mathrm{Bcl}-\mathrm{xL}$ was expressed by many cell types, including lymphocytes and epithelial cells (Supplemental Figure 3A). Therefore, to analyze $\mathrm{Bcl}-\mathrm{xL}$ expression by IELs, lymphocytes were isolated from duodenal biopsies, and intracellular expression of Bcl-xL was studied by flow cytometry. Whereas few IELs were positive in controls (median, 13\%; range, 1\%-20\%), the majority of IELs expressed Bcl-xL in RCDII (median, 59\%; range, 42\%-97\%), ACD (median, $51 \%$; range, $15 \%-93 \%$ ), and GFD (median, $53 \%$; range, $30 \%-$ $73 \%)$. In contrast, there was no significant difference in $\mathrm{Bcl}-\mathrm{xL}$ expression by lamina propria lymphocytes (LPLs) between CD patients and controls (Figure 6B).

Immunohistochemical staining indicated that, in contrast to $\mathrm{Bcl}-\mathrm{xL}$, expression of $\mathrm{Bcl}-2$ in the intestine was restricted to lymphocytes (Supplemental Figure 3B). Surprisingly, Bcl-2 was decreased in IELs from ACD or RCDII patients compared with controls and patients on a GFD. Immunoblotting demonstrated reduced amounts of $\mathrm{Bcl}-2$ protein in duodenal extracts from ACD and RCDII patients compared with patients on a GFD $(P<0.05$, Mann Whitney test; Figure 6A). The difference did not reach statistical significance compared with controls, likely due to the massive increase in IEL numbers in ACD and RCDII. Indeed, the percent- 

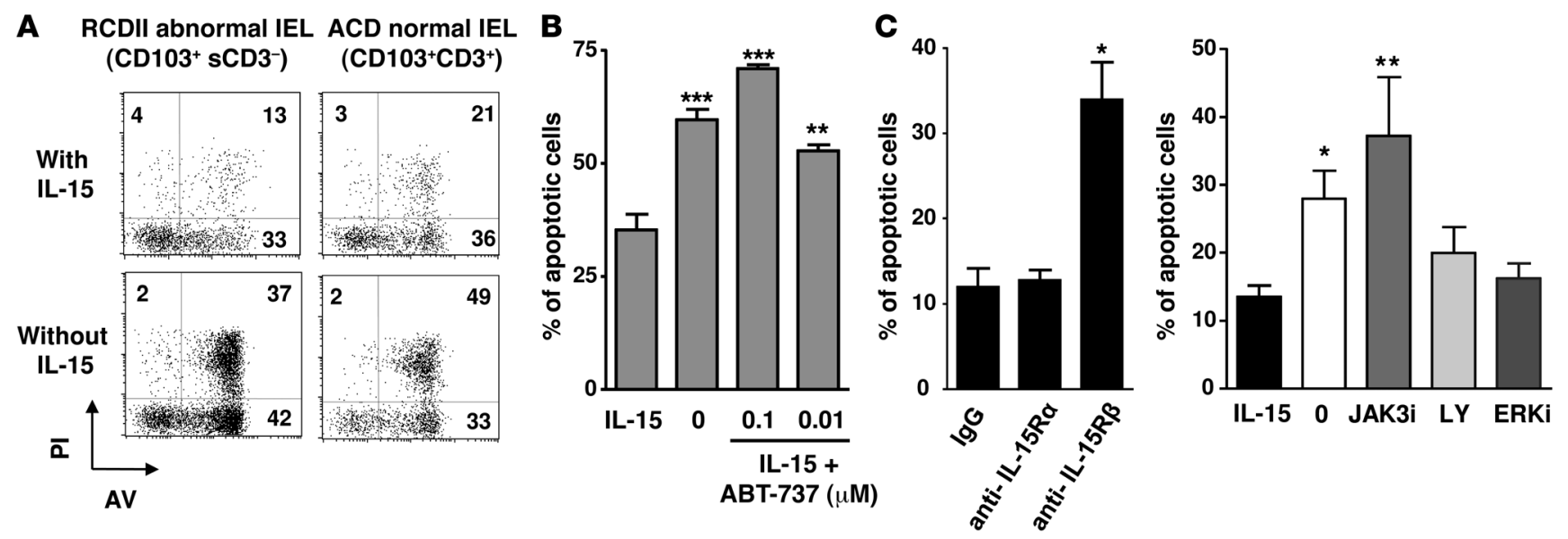

Figure 4

IL-15 activates the same antiapoptotic signaling pathway in RCDII and normal IELs from control and ACD patients. (A) Freshly isolated IELs from RCDII or ACD patients labeled with annexin V/PI after a 72-hour culture in the presence or absence of IL-15. All cells were protected from apoptosis by IL-15. (B and C) Percent of apoptotic cells, determined as in Figure 2A, after a 48-hour culture of normal IEL lines $(n=4)$ in the presence or absence of 5-10 ng/ml IL-15 and (B) ABT-737 at the indicated concentrations or (C) blocking anti-IL-15R $\alpha$ or -IL-15R $\beta$ mAbs and control isotype or pharmacological inhibitors. ${ }^{\star} P<0.05,{ }^{\star \star} P<0.01,{ }^{\star \star \star} P<0.001$ versus respective control, 1 -way ANOVA with Tukey's multiple comparison test.

age of Bcl-2+ IELs in tissue sections or in freshly isolated IELs was drastically reduced in ACD and RCDII patients compared with controls and fell between control and ACD values in patients on a GFD (Figure 6B). A decrease in the percentage of Bcl-2+ $\mathrm{T}$ cells, albeit much more moderate, was also observed in LPLs (Figure 6B), but no difference between patients and controls was detected in peripheral blood (data not shown). Thus, freshly isolated abnormal IELs from RCDII did not express Bcl-2, in contrast to both residual normal IELs and IELs from controls (Supplemental Figure 3C). Therefore, Bcl-xL, but not Bcl-2, might stimulate the survival and accumulation of IELs in ACD and RCDII.

Expression of phospho-Jak3 and phospho-STAT5 is increased in intestinal lymphocytes in RCDII and ACD and is downmodulated by a blocking anti-IL-15 Ab. Because phosphorylation of STAT5 was labile and disappeared after a 30 -minute incubation at $37^{\circ} \mathrm{C}$ (data not shown), it was not possible to analyze phospho-STAT5 in freshly isolated IELs and LPLs by flow cytometry. In situ expression of phospho-STAT5 and phospho-Jak3 was therefore studied by immunohistochemical staining of formalin-fixed duodenal biopsies. As shown in Figure 7, control biopsies contained a moderate number of phospho-Jak3 ${ }^{+}$and phospho-STAT5 $5^{+}$intestinal lymphocytes preferentially localized in the epithelium and in the upper part of the villi. In ACD and in RCDII, there was a dramatic increase in the number of phospho-Jak $3^{+}$and phospho-STAT5 ${ }^{+}$ lymphocytes localized in both the epithelium and the lamina propria (Figure 7, A and B). Phospho-Jak3 ${ }^{+}$and phospho-STAT5 ${ }^{+}$lymphocytes remained more numerous in biopsies from patients on a GFD (data not shown) than in those from controls, a finding consistent with the observation that Bcl-xL expression by IELs did not return to control levels after GFD (Figure 6A). Jak3 can be activated by IL-15, but also by several other cytokines, some of which might be upregulated in CD. Therefore, to evaluate the role of IL-15 in the local activation of the Jak3/STAT5 pathway, duodenal biopsies from RCDII patients were cultured for 48 hours in the presence of the fully humanized anti-human IL-15 mAb AMG714. Expression of phospho-Jak3 and phospho-STAT5 was reduced in biopsies treated with AMG714 compared with biopsies cultured with control IgG (Figure 7, C and D, and Supplemental Figures 5 and 6), which indicates that local synthesis of IL-15 participates in activation of Jak3 and STAT5. In addition, Bcl-xL expression was downregulated by AMG714 (Figure 7D). Collectively, these data support the hypothesis that blocking IL-15 might be useful to inhibit the survival and thereby the accumulation of normal IELs in ACD and, more importantly, of transformed IELs in RCDII.

AMG714 treatment of mice overexpressing human IL-15 in their gut epithelium restores intestinal lymphocytes' apoptosis and reduces their massive accumulation. AMG714 has recently been used without severe side effects in phase I and II clinical trials for rheumatoid arthritis (30). We tested its capacity to reverse the abnormal in vivo survival and accumulation of IELs in C57BL/6 (B6) mice overexpressing human IL-15 under the control of T3b, an enterocyte-specific promoter (IL-15Tg $\mathrm{E}_{\mathrm{E}}$ mice; ref. 31). Consistent with previous observations (31), IL-15 $\mathrm{Tg}_{\mathrm{E}}$ mice exhibited a massive expansion of IELs compared with B6 littermates $\left(7 \pm 1.25 \times 10^{6}\right.$ vs. $2.0 \pm 0.5 \times 10^{6}$; $P=0.02$, Mann-Whitney test; Figure 8, A and B). As in ACD and RCDII patients, the vast majority of IELs - if not all - failed to express the KI67 proliferation marker, which suggests that their accumulation was the result of impaired apoptosis (Figure 8A). Accordingly, IELs from IL-15Tg mice, but not from B6 littermates, demonstrated high expression of phospho-Jak3 and phospho-STAT5 (data not shown). Treatment of IL- $15 \mathrm{Tg}_{\mathrm{E}}$ mice with AMG714, but not with a control human monoclonal IgG, increased apoptosis in IELs and LPLs within 48 hours of the first Ab injection (Figure 8C) and drastically decreased the number of IELs to levels seen in B6 littermates after 2 weekly injections (AMG714, $1.7 \pm 0.25$ $\times 10^{6} ; \mathrm{B} 6,2.0 \pm 0.5 \times 10^{6} ; P=0.01$; Figure $\left.8 \mathrm{~B}\right)$.

Treatment with $A B T-737$ restores normal numbers of $C D 8^{+} T, N K$, and NKT cells in the blood of $I L-15 \mathrm{Tg}_{E}$ mice. To confirm in vivo the role of $\mathrm{Bcl}-2$ and Bcl-xL in IL-15-mediated survival, we next treated IL-15TgE and $\mathrm{B} 6$ control mice with ABT-737. Basal numbers of CD8 ${ }^{+} \mathrm{T}, \mathrm{NK}$, and NKT cells were markedly increased in the blood of IL-15Tg mice compared with B6 controls (Figure 9A), a result in keeping with the presence of large amounts of serum human IL-15 released by intestinal epithelial cells, which express a secreted form of human 

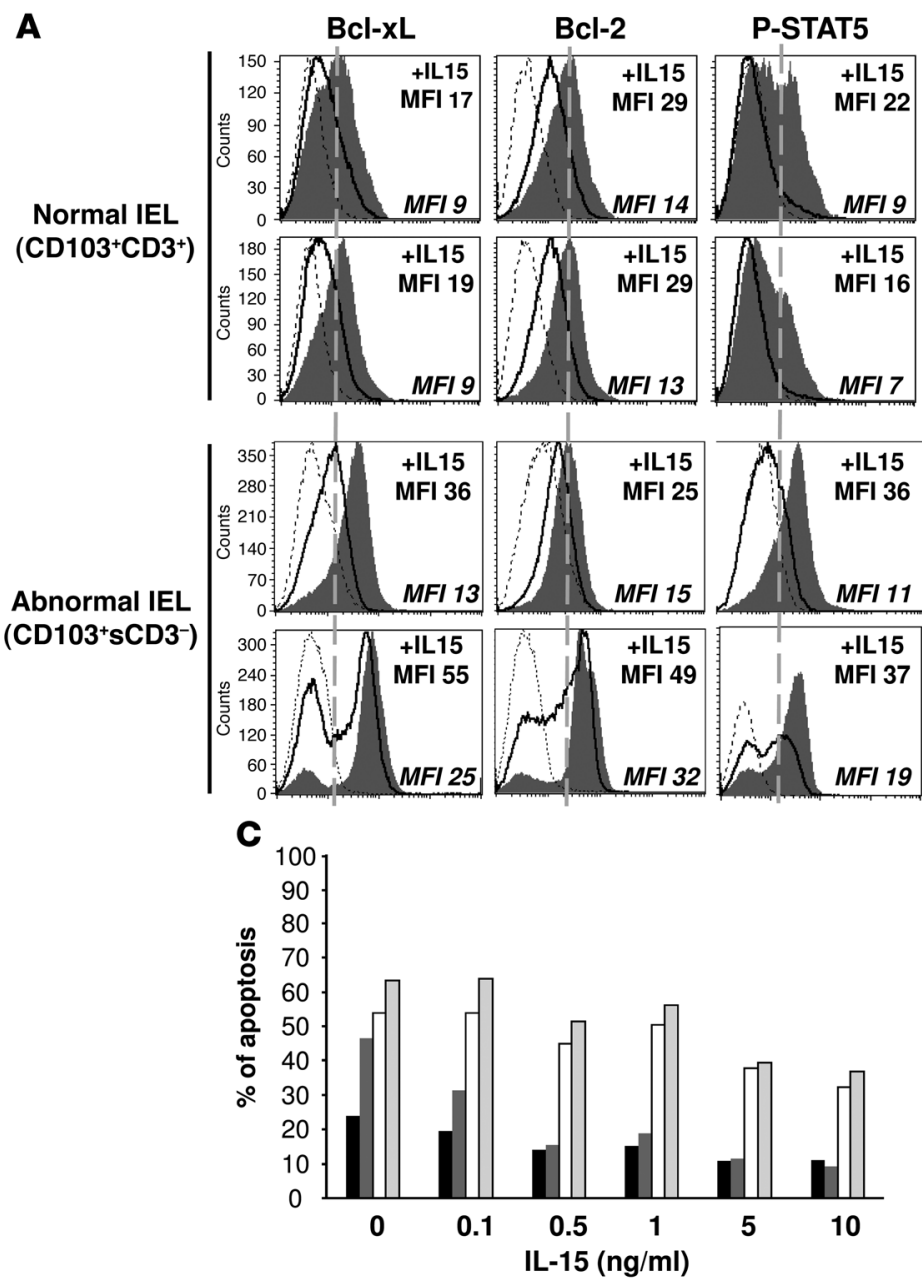
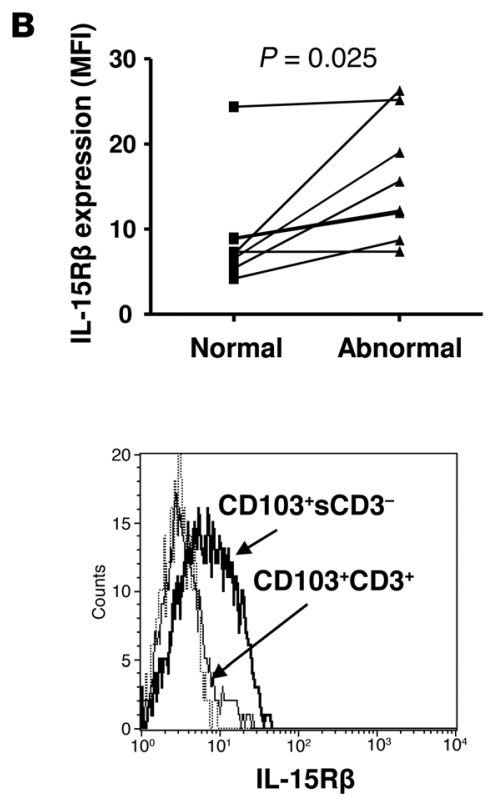

RCDII IEL line 1

RCDII IEL line 2

CT IEL line 1

CT IEL line 2

\section{Figure 5}

RCDII IELs are more sensitive to IL-15 antiapoptotic signaling than are normal IELs. (A) Flow cytometry analysis of Bcl-2, Bcl-xL, and phosphoSTAT5 in 2 normal and 2 RCDII IEL lines cultured in the absence (black histograms) or presence of $5 \mathrm{ng} / \mathrm{ml} \mathrm{IL-15}$ (gray filled histograms). MFI was higher in RCDII than in normal IEL lines. Dashed lines denote isotype controls. (B) Flow cytometry analysis of IL-15R $\beta$ (CD122) in freshly isolated IELs from 8 RCDII patients. MFI of CD122 significantly increased in abnormal IELs compared with residual normal IELs (paired $t$ test). Below, representative FACS staining of freshly isolated IELs from 1 RCDII patient showed higher expression of CD122 on gated abnormal CD103+SCD3- IELs compared with normal residual CD3+CD103+ IELs. (C) Flow cytometry analysis of apoptosis in normal control (CT) and RCDII IEL lines after a 72-hour culture with the indicated concentrations of IL-15. Rescue from apoptosis required lower IL-15 concentrations in RCDII than in control IEL lines.

IL-15 (31). Treatment of IL-15Tg mice with ABT-737 resulted in the same drastic reduction in these 3 populations and restored levels observed in $\mathrm{B} 6$ control mice after 7 days of treatment (Figure 9A). Notably, the effect of ABT-737 on CD8 ${ }^{+} \mathrm{T}, \mathrm{NK}$, and NKT cells was comparable to that of AMG714 (Figure 9B), which indicates that ABT-737 acted preferentially on these IL-15-dependent populations. ABT-737 treatment also restored normal levels of $\mathrm{T}$ cells in the spleens and mesenteric lymph nodes of IL-15Tg $\mathrm{E}_{\mathrm{E}}$ mice (Figure 9C). Treatment with ABT-737 i.p. had little or no effect on LPLs and IELs (Figure 9C), although it efficiently blocked the antiapoptotic effect of IL-15 on murine IELs cultured for 48 hours in vitro (Figure 9D).

\section{Discussion}

Combining in vitro, ex vivo, and in situ approaches, we showed that IL-15 activated an antiapoptotic cascade involving the $\beta \gamma_{c}$ module of the IL-2/IL-15 receptor, phosphorylation of Jak3 and STAT5, and expression of Bcl-xL in either normal or transformed RCDII IELs. We further observed that AMG714, a humanized anti-IL-15 $\mathrm{mAb}$, inhibited endogenous activation of Jak 3 and STAT5 in intestinal organ cultures from ACD and RCDII and, in vivo, stimulated IEL apoptosis and drastically reduced the massive IEL infiltration characteristic of IL-15Tg $\mathrm{Tg}_{\mathrm{E}}$ mice, which overexpress human IL-15 in their gut epithelium. Together, these results provide a rationale for a strategy consisting of blocking IL-15 antiapoptotic signals to eliminate malignant IELs in RCDII patients, avoid their diffusion to the periphery, and prevent their almost-inevitable transformation into aggressive lymphomas.

Other than activation of $\mathrm{CD}^{+}$lamina propria $\mathrm{T}$ lymphocytes by gluten peptides presented by HLA-DQ2 or -DQ8, one cardinal feature of CD is the loss of IEL homeostasis, causing massive accumulation of activated IELs that subsides only partially after GFD and emergence of transformed IELs in a small 

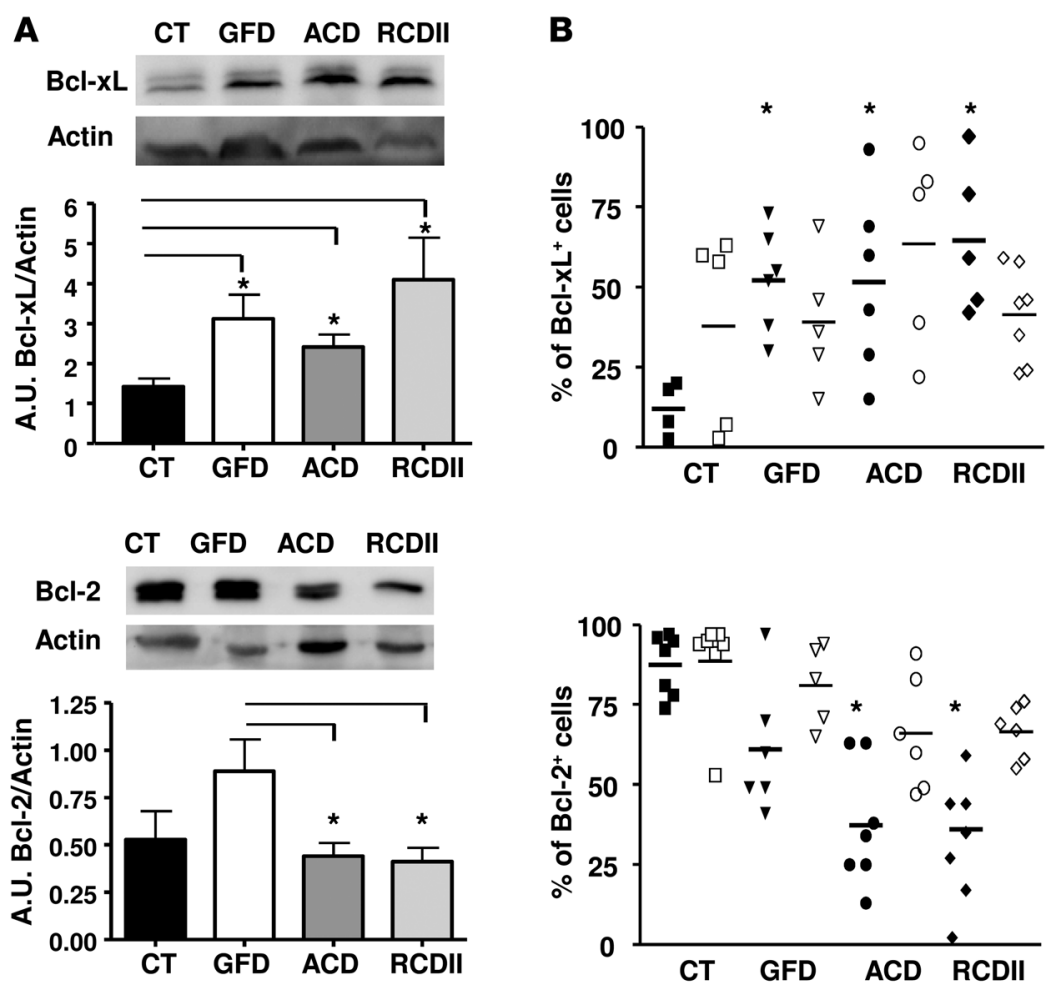

\section{Figure 6}

Increased expression of $\mathrm{Bcl}-\mathrm{xL}$, but not $\mathrm{BCl}-2$, in $\mathrm{ACD}$ and RCDII IELs. (A) Western blot analysis of $\mathrm{Bcl}-\mathrm{xL}$ and $\mathrm{Bcl}-2$ in duodenal extracts from 5 controls, $4 \mathrm{CD}$ patients on GFD, 6 ACD patients, and 5 RCDIl patients. ${ }^{*} P<0.05$. (B) Flow cytometry analysis of $\mathrm{Bcl}-\mathrm{xL}$ and $\mathrm{Bcl}-2$ in freshly isolated IELs (closed symbols) and LPLs (open symbols). $\mathrm{Bcl}-\mathrm{xL}$ expression significantly increased in all CD patients, whereas $\mathrm{Bcl}-2$ significantly decreased in ACD and RCDII patients, in IELs but not in LPLs. ${ }^{\star} P<0.05$ versus control, Mann-Whitney test.

subset of patients that fail to respond to GFD. Because neither nontransformed IELs in CD nor transformed IELs in RCDII express the proliferation marker KI67, we have previously suggested that IEL accumulation is not caused by local proliferation, but rather results from an antiapoptotic signal delivered by IL-15 overexpressed in the gut epithelium (6). The massive accumulation of $\mathrm{KI}^{6} 7^{-}$IELs in $\mathrm{IL}-15 \mathrm{Tg}_{\mathrm{E}}$ mice is consistent with this hypothesis. The antiapoptotic role of IL-15 is firmly established, but the subsequent signaling cascade remains debated $(16,17)$. In mice, both Il15ra-/- (32) and Il15rb-/- (33) mice have IEL defects, which indicates that both chains of the IL-15 receptors are involved in IEL homeostasis. A direct role of IL-15R $\alpha$ in the delivery of an antiapoptotic signal was suggested by some in vitro studies (reviewed in ref. 16). Yet in most mouse in vivo studies, IL-15R $\alpha$ does not seem to be part of the antiapoptotic signaling machinery, but rather appears as a ubiquitous chaperone molecule that is able to stabilize IL-15 and promotes its transpresentation to lymphocytes via the $\beta \gamma_{c}$ signaling module common to IL-15 and IL-2 receptors (16). Here we have shown that blocking IL-15R $\beta$, but not IL-15R $\alpha$, inhibited the antiapoptotic signal delivered in vitro by soluble IL-15 to normal and abnormal IEL lines, demonstrating that signal transduction exclusively depended on the $\beta \gamma_{c}$ module. IL-15R $\alpha$, which is upregulated in the intestinal mucosa of CD patients, might stabilize IL-15 and promote its presentation to intestinal lymphocytes (34).
IL-2/IL-15R $\beta \gamma_{c}$ activates the PI3K/AKT, ERK, and JAK/STAT signaling pathways, all previously shown to be involved in IL-15-driven lymphocyte survival. Several recent studies have emphasized a central role of PI3K through the modulation of proapoptotic factors. In mouse NK cells, IL-15 inhibited BIM expression via PI3K-dependent inactivation of the transcription factor Foxo3a. In addition, IL-15 stimulated proteasome-mediated degradation of BIM by activating Erk1 and Erk2 (18). In humans, IL-15-dependent activation of PI3K and p38 was previously shown to stimulate proteasome degradation of BID in normal NK cells and in large granular $\mathrm{T}$ and NK leukemic lymphocytes (35). In RCDII lines, BID expression either did not increase or increased modestly after IL-15 starvation (Supplemental Figure 2). Furthermore, the PI3K inhibitor LY294002, which strongly induced BID in RCDII IEL lines, failed to induce their apoptosis, arguing against a critical role for this proapoptotic factor, at least when $\mathrm{Bcl}-\mathrm{xL}$ and/or Bcl-2 is strongly expressed.

In contrast, our results demonstrated that IL-15driven survival of IELs depended on Jak3 activation. Jak3 plays a central role in the early phases of lymphocyte development mainly ascribed to its contribution to IL-7R signaling. Yet treatment of adult animals and of transplanted patients with pharmacological inhibitors of Jak3 induce depletion in mature $\mathrm{NK}$ and memory $\mathrm{CD}^{+} \mathrm{T}$ cells (reviewed in ref. 36), resulting in a phenotype reminiscent of $I l 15^{-/-}$animals. Interestingly, a comparable phenotype has been observed in mice with selective inactivation of STAT $5 \mathrm{a} / \mathrm{b}$ in mature lymphocytes $(37,38)$. Through Jak3, IL-15 can activate the transcription factors STAT5 and STAT3, both of which regulate the expression of antiapoptotic genes and have oncogenic properties $(39,40)$. Using shRNA, we observed that STAT5, but not STAT3, supported IL-15-mediated survival of RCDII IELs. Confirming the hypothesis that IL-15 might drive the survival of IELs through the Jak3/STAT5 pathway in vivo in CD and RCDII, in situ analysis revealed increased expression of phospho-Jak3 and phospho-STAT5 in intestinal lymphocytes of ACD and RCDII patients. Furthermore, in situ activation of Jak3 and STAT5 was dependent on IL-15, since adding a blocking anti-IL-15 mAb in duodenal organ cultures from ACD and RCDII patients downmodulated their phosphorylation.

STAT5 has a binding site on the Bcl-xL promoter and can upregulate $\mathrm{Bcl}-\mathrm{xL}(41,42)$. Analysis of STAT5 null versus STAT5 transgenic mice also demonstrated its role in driving Bcl-2 expression in lymphocytes (37). In contrast, STAT3 was shown to control survival of large granular leukemic lymphocytes through Mcl-1 upregulation (43). We found that apoptosis induction in response to STAT5 inhibition by shRNA or IL-15 withdrawal was associated with decreased expression of Bcl-2 and an even more dramatic decrease in Bcl-xL, but had no or little effect on Mcl-1, which suggests that Mcl-1 is not sufficient to drive IEL survival. The latter finding was confirmed by showing that ABT-737, a drug that blocks the antiapoptotic effect of Bcl-2 and Bcl-xL but not of Mcl-1 (24), inhibited IL-15 prosurvival signals in normal and 
A
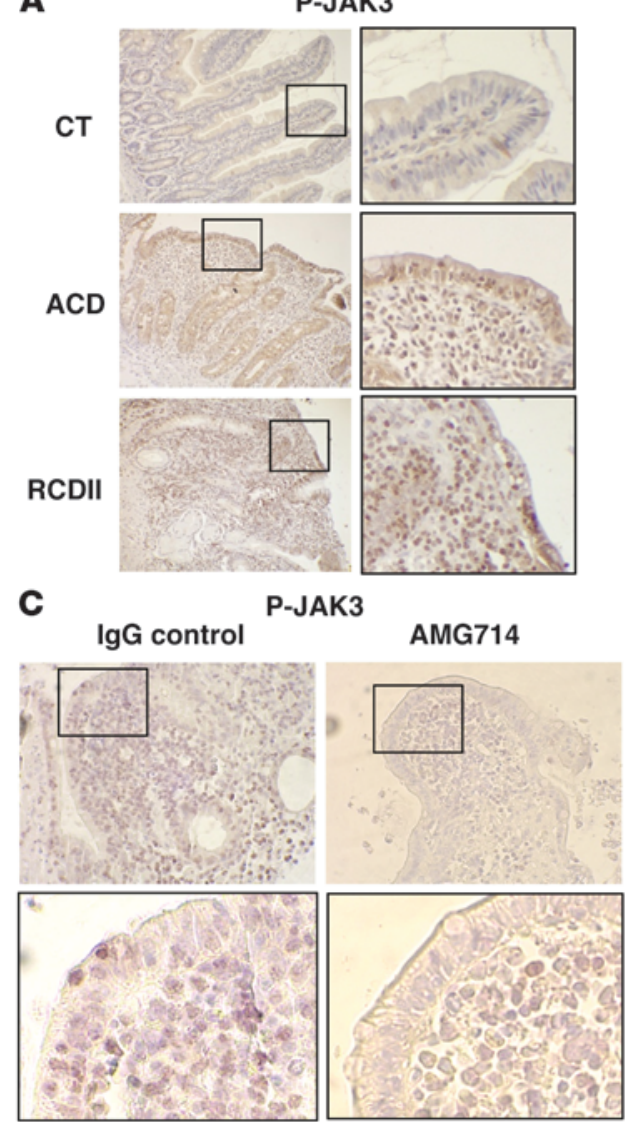

B
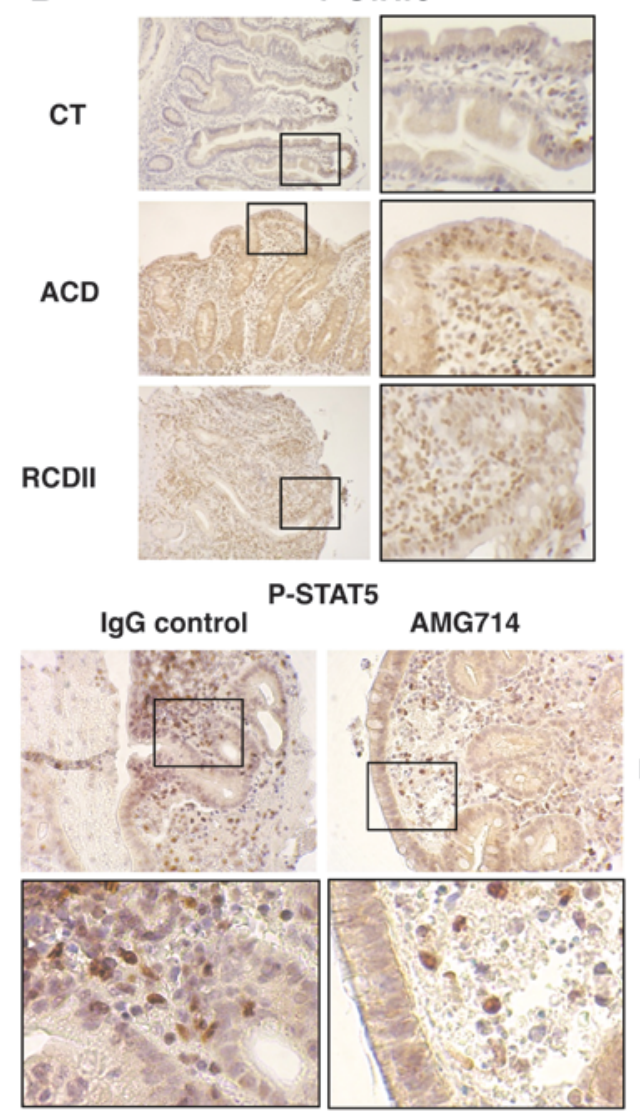

D

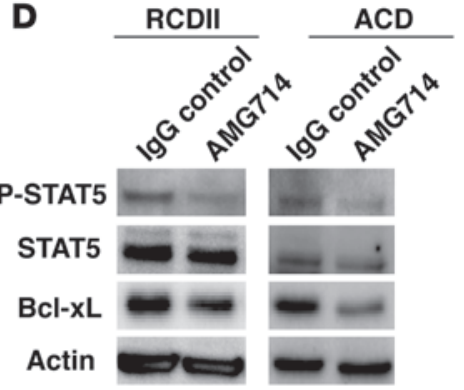

Figure 7

IL-15-dependent phosphorylation of Jak3 and STAT5 in RCDII and ACD. (A and B) Representative immunohistochemical staining of duodenal biopsies from control $(n=4)$, ACD $(n=5)$, and RCDII $(n=4)$ individuals with anti-phospho-Jak3 (A) or anti-phospho-STAT5 (B) Abs. (C) Immunostaining of duodenal biopsies from 1 RCDII patient with anti-phospho-Jak3 or anti-phospho-STAT5 Abs after a 48-hour organ culture in the presence of $10 \mu \mathrm{g} / \mathrm{ml}$ AMG714 or control IgG. Shown is 1 representative of 4 experiments. (A-C) Panels with outlines show higher-magnification views of the boxed regions in the adjacent images. Original magnification, $\times 10 ; \times 40$ (higher magnification). (D) STAT5 phosphorylation and $\mathrm{Bcl}-\mathrm{xL}$ expression were investigated using Western blot in biopsies from 1 RCDII and 1 ACD patient cultured for 48 hours with AMG714 or control lgG.

transformed IELs. Similar results were obtained in human primary NK $\left(\mathrm{CD}^{-}{ }^{-} \mathrm{CD} 56^{+}\right)$and $\mathrm{CD}^{+} \mathrm{T}$ cell lines (data not shown), suggesting that, in contrast to its role in mouse NK cells (18), Mcl-1 might not be essential for IL-15-mediated survival in human lymphocytes. Previous studies have also suggested a role for Bcl-2 and Bcl-xL in IL-15-mediated survival of NK cells $(19,20,26,44), \mathrm{CD}^{+}$ $\mathrm{T}$ cells (45-47), and IELs (48). Our ex vivo and in situ analysis of IELs showed upregulation of Bcl-xL in ACD and RCDII, whereas surprisingly, Bcl-2 expression was diminished compared with controls and patients on a GFD. This result suggests that Bcl-xL, more than Bcl-2, might drive IL-15-dependent survival of IELs in the inflamed intestines of patients with ACD and RCDII. Interestingly, it was recently shown that trans-presentation of IL-15 via IL-15R $\alpha$ induces expression of Bcl-xL, but not Bcl-2, in NK cells (26). Because IL-15R $\alpha$ is upregulated in epithelium and lamina propria of CD patients (34), one may speculate that IL-15/IL-15R $\alpha$ complexes selectively stimulate Bcl-xL expression. Alternatively, local inflammation might inhibit Bcl-2 expression in lymphocytes. Indeed, activation through the TCR $(49,50)$ or reactive oxygen species generated during inflammation (51) were previously shown to downmodulate Bcl-2 in T cells. Consistent with the latter hypothesis, we found that $\mathrm{Bcl}-2$ expression was almost normal in CD patients on a GFD. Bcl-xL is mainly expressed by activat$\mathrm{ed} /$ memory lymphocytes $(45,49)$. Since IL-15 is upregulated in response to infections, IL-15-induced upregulation of Bcl-xL may help effector lymphocytes to escape activation-induced cell death in infected tissues and thereby help eliminate the causative pathogen. Yet, as illustrated here in CD, protracted production of IL-15 might result in accumulation of proinflammatory and/or autoreactive lymphocytes sustaining chronic inflammation and autoimmunity $(6,52,53)$. Using RCDII as a unique model to analyze the first steps of $\mathrm{T}$ cell lymphomagenesis in humans, we provide striking evidence that amplification of a normal antiapoptotic signaling pathway can promote the accumulation of clonal transformed T lymphocytes. Increased expression of IL-2/IL-15R $\beta \gamma_{c}$ on transformed RCDII IELs compared with residual normal IELs may enable them to respond to lower concentrations of IL-15, providing them with a selective advantage that could explain their progressive accumulation and the simultaneous disappearance of normal IELs. It remains to be determined whether high expression 
A

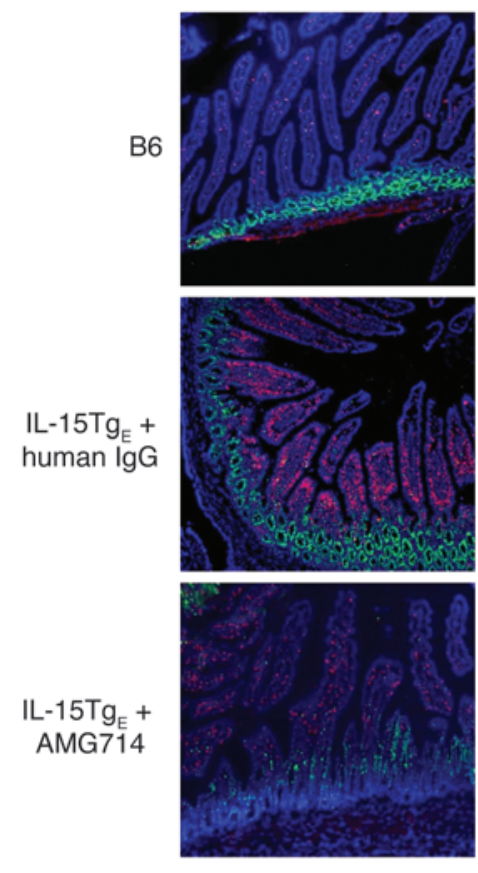

B

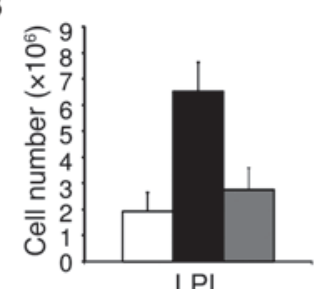

C

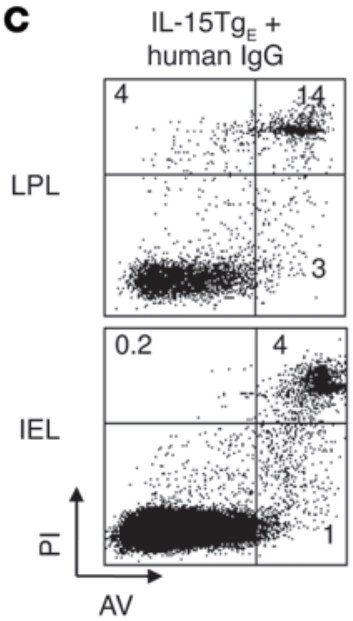

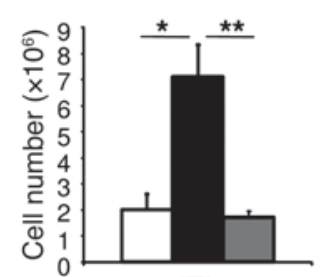

IEL

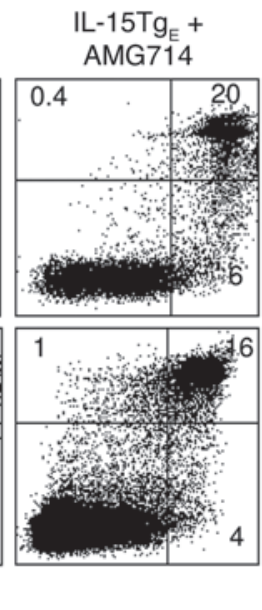

\section{Figure 8}

Treatment of IL-15TgE mice with AMG714 restores apoptosis of intestinal lymphocytes and inhibits their accumulation. (A) Frozen duodenal sections of IL-15TgE mice and B6 littermates labeled with anti-CD3 (red) and anti-KI67 (green) Abs. In IL-15TgE mice, intestinal accumulation of $\mathrm{CD}^{+}{ }^{+} \mathrm{KI} 67^{-}$lymphocytes (middle) suggested impaired lymphocyte apoptosis. Numbers of CD3+ lymphocytes decreased markedly after 2 weekly i.p. injections of $50 \mu \mathrm{g}$ AMG714, but not of control human IgG. (B) Flow cytometry determination, using Trucount beads, of CD3+ lymphocyte number in LPLs and IELs from B6 and IL-15TgE mice. Treatment with AMG714 (as in A), but not with control IgG, reduced IEL numbers to those observed in B6 littermates. ${ }^{\star} P<0.5,{ }^{\star \star} P<0.01$, Mann-Whitney. (C) Flow cytometry analysis demonstrated increased apoptosis of IELs and LPLs in IL-15Tg $\mathrm{T}_{\mathrm{E}}$ mice 2 days after 1 i.p. injection of AMG714. Shown is 1 representative of 3 experiments.

of IL-2/IL-15R $\beta \gamma_{c}$ by RCDII IELs is related to their origin from the small normal subset of IL-2/IL-15R $\beta \gamma_{c}$-high IELs deprived of surface CD3-TCR complexes, to a genetic aberration, or to an acquired event induced in the local inflammatory environment.

Because activation of Jak3 and STAT5 in RCDII remains dependent on IL-15, a therapeutic approach based on the neutralization of the activating cytokine or of its receptor might be useful to eliminate abnormal cells at an early stage of lymphomagenesis, before they acquire new genetic aberrations and transform into an overt lymphoma. As a first step to test this concept, we demonstrated that AMG714 restored IEL apoptosis, thereby reducing the massive accumulation of IELs in transgenic mice overexpressing human IL-15 in their gut epithelium (31). Yokoyama et al. have recently observed that an IL-2/IL-15R $\beta$ Ab can reverse the inflammatory epithelial lesions that develop in this model after 6-8 months of age (54). Together, these data indicate that blocking IL-15 signaling may both alleviate epithelial damage and eradicate transformed IELs in RCDII. Interestingly, treatment of IL-15Tg $\mathrm{T}_{\mathrm{E}}$ mice with ABT-737, like treatment with AMG714, reduce the expansion of circulating $\mathrm{CD}^{+} \mathrm{T}$, NK, and NKT cells, which suggests that these 3 IL-15-dependent populations rely on $\mathrm{Bcl}-2$ and/or Bcl-xL for their survival. These results contrast with a previous publication indicating that IL-15 mediates survival of NK cells in mice through the expression of Mcl-1 (17), but are consistent with other studies
$(19,20,26,44)$. In contrast to AMG714 therapy, treatment with ABT-737 failed to restore normal homeostasis of LPLs and IELs in IL-15Tg $\mathrm{g}_{\mathrm{E}}$ mice. Because ABT-737 efficiently blocked the antiapoptotic effect of IL-15 on murine IELs in vitro and restored normal homeostasis of circulating $\mathrm{CD}^{+} \mathrm{T}, \mathrm{NK}$, and NKT cells, distribution of this $\mathrm{Bcl}-2 / \mathrm{Bcl}-\mathrm{xL}$ inhibitor might not be optimal in the intestine. Beyond RCDII, therapeutic strategies based on inhibition of IL-15 or its downstream antiapoptotic signaling pathway might be useful to treat inflammatory or autoimmune disorders associated with protracted expression of IL-15 and accumulation of activated $\mathrm{CD} 8^{+} \mathrm{T}$ cells and/or NK cells, if their distribution can be achieved in the target tissue.

\section{Methods}

Patients and controls. Endoscopic biopsies were obtained from CD patients. Patients with ACD ( $n=13$; mean age, 41 years) were diagnosed based on clinical symptoms, positive serology for antitissue transglutaminase Abs, and partiality to subtotal villous atrophy on normal diet. Treated CD patients were on GFD for at least 1 year $(n=10$; mean age, 43 years); they had recovered normal or almost-normal villous architecture and a negative serology. RCDII ( $n=14$; mean age, 52 years) was defined as described previously $(3,4)$ : by malabsorption and villous atrophy persistent after 1 year of strict adherence to GFD, associated with greater than $30 \% \mathrm{CD}_{103}{ }^{+} \mathrm{SCD} 3^{-}$IELs (as assessed by flow cytometry phenotyping of fresh IELs isolated from biopsies) and clonal TCR $\gamma$ 
A
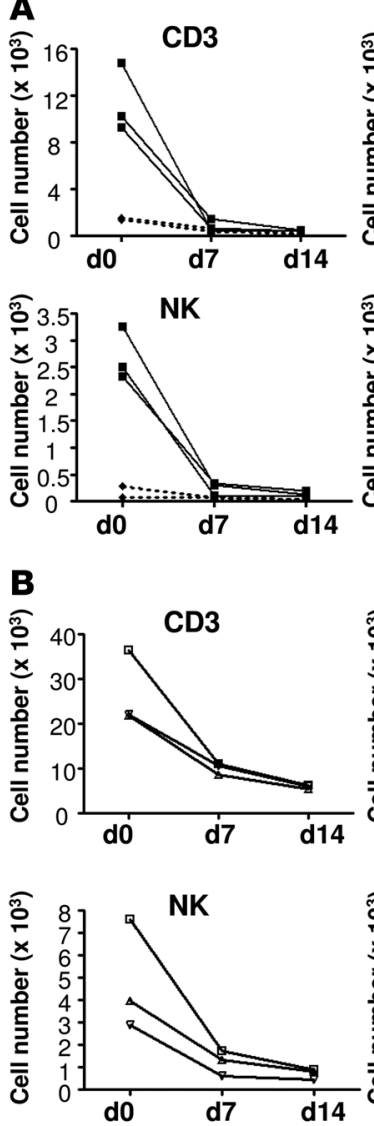
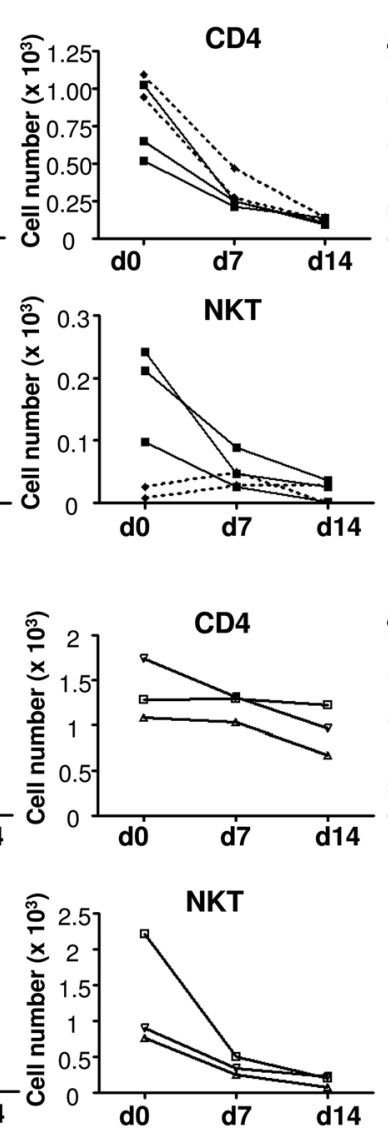
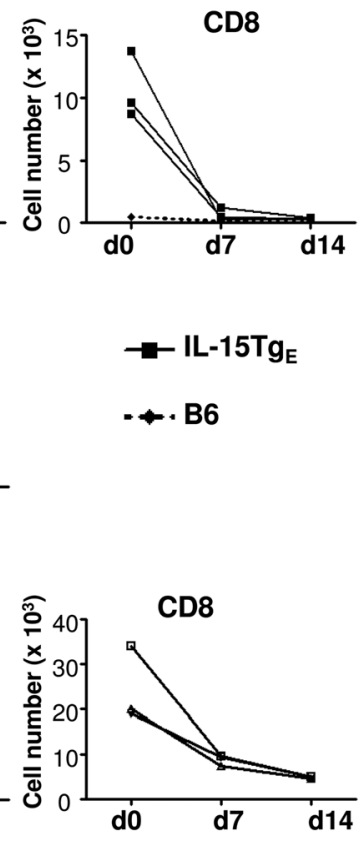

$\rightarrow-I L-15 T g_{E}$ $\because$ - B6

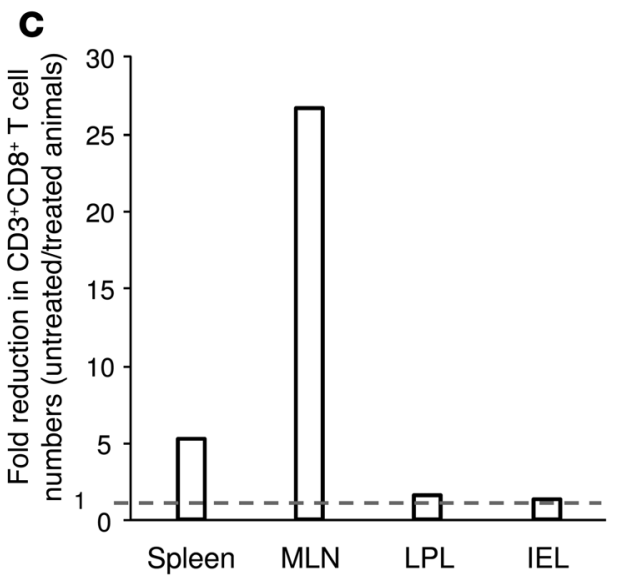

D

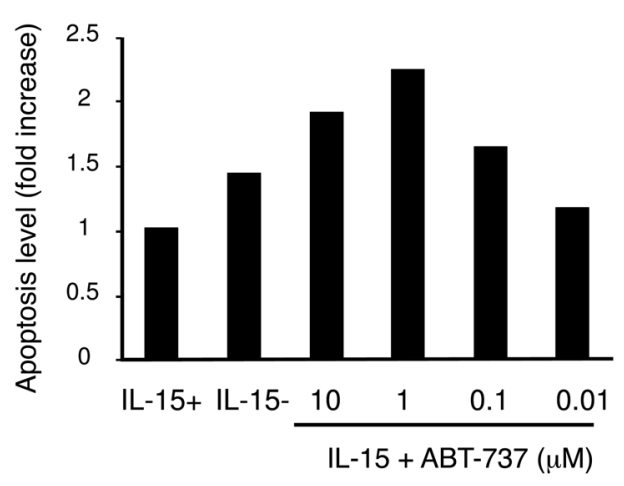

Figure 9

Treatment of IL-15TgE mice with ABT-737 restores normal numbers of circulating CD8 ${ }^{+} \mathrm{T}$, NK, and NKT cells. (A) 3 IL-15TgE and 2 B6 mice were treated with daily i.p. injections of $75 \mathrm{mg} / \mathrm{kg} \mathrm{ABT}-737$ or vehicle, and absolute numbers of CD8 ${ }^{+} \mathrm{T}$, NK, and NKT cells were determined in the blood on days 7 and 14. Treatment of IL-15TgE mice markedly decreased the number of circulating CD8 ${ }^{+} T$, NK, and NKT cells, which returned to that observed in B6 mice. No changes in the numbers or percentages of cells were observed in mice that received vehicle only (not shown). (B) For comparison, IL-15TgE mice were treated with 2 i.p. injections of AMG714 or control human IgG ( $n=3$ per group). AMG714, like ABT-737, preferentially decreased the number of CD8 ${ }^{+} \mathrm{T}$, NK, and NKT cells in the blood of IL-15TgE mice, whereas control human IgG had no effect (not shown). (C) Treatment of IL-15Tg $\mathrm{F}_{\mathrm{E}}$ mice with ABT-737 induced a marked decrease of CD3 ${ }^{+} \mathrm{CD} 8^{+} \mathrm{T}$ cells in spleen and mesenteric lymph nodes (MLNs), but had little or no effect on LPLs and IELs, compared with mice treated with vehicle. (D) IELs isolated from IL-15TgE mice were cultured in vitro for 48 hours in medium alone and supplemented with $20 \mathrm{ng} / \mathrm{ml} \mathrm{IL-15}$ in the presence of different concentrations of ABT-737. Results are representative of 2 independent experiments.

and/or TCR $\delta$ rearrangements in duodenal biopsies detected by multiplex PCR. All RCDII patients were HLA-DQ2 and had exhibited celiac Abs (serum IgA and/or IgG anti-gliadin Abs, IgA class endomysial Abs, and/or anti-human IgA tissue transglutaminase Abs) prior to GFD. 7 patients $(50 \%)$ had initially responded to GFD (mean gluten responsiveness time for all patients, 35 months; range, 0-117 months). The percent $\mathrm{CD}_{103}{ }^{+} \mathrm{SCD}^{-}$- IELs was $83 \%$ (range, 54\%-98\%). Histologically normal intestinal samples (controls), provided by P. Wind (Hôpital Avicenne, Bobigny, France) and A. Berger (Hôpital Européen Georges Pompidou, Paris, France), had been obtained from patients undergoing intestinal surgery for morbid obesity or pancreatic cancer $(n=8$; mean age, 58 years). All studies using human subjects were reviewed and approved by the Ethics committee Ile-de-France II (Paris, France). Patients provided informed consent prior to their participation in this study.

Cell isolation, cell lines, and cell culture. IELs and LPLs were isolated as described previously $(13,55)$ and cultured in RPMI with $10 \%$ AB human serum supplemented with $1 \%$ sodium pyruvate, $1 \%$ nonessential amino acids, $1 \%$ HEPES buffer, $40 \mu \mathrm{g} / \mathrm{ml}$ gentamicin, and $5 \times 10^{-5} \mathrm{M} \beta$-mercapto- ethanol (Invitrogen). CD103+sCD3- IEL lines were derived from duodenal biopsies from RCDII patients as described previously (6) in medium containing $20 \mathrm{ng} / \mathrm{ml}$ human IL-15 (R\&D Systems).

For analysis of IL-15 antiapoptotic signaling, IEL lines or fresh IELs were cultured for 48-96 hours with $5 \mathrm{ng} / \mathrm{ml} \mathrm{IL-15}$ in the presence of $100 \mu \mathrm{M}$

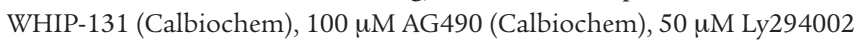
(Sigma-Aldrich), $500 \mathrm{nM}$ wortmannin (Sigma-Aldrich), $10 \mu \mathrm{M}$ U0126 (Sigma-Aldrich), indicated concentrations of ABT-737 (obtained from Abbott Laboratories Inc.), or $10 \mu \mathrm{g} / \mathrm{ml} \mathrm{mAbs} \mathrm{blocking} \mathrm{human} \mathrm{IL-15R} \alpha$ (M165; gift from AMGEN Inc.) or IL-15R $\beta$ (clone A41 mAb; gift from Y. Jacques, INSERM U601, Nantes, France).

For organ culture, duodenal biopsies from RCDII or ACD patients were cultured as described previously (6) in the presence of $10 \mu \mathrm{g} / \mathrm{ml} \mathrm{AMG714}$ or a control human monoclonal IgG1к (clone $120.6 .1 \mathrm{G} 1 \mathrm{k}$; both gifts from AMGEN Inc.) for 48 hours.

shRNA. Sense and antisense oligonucleotides covering nucleotide $+1,581$ to $+1,599$ from Stat5a initiation codon were annealed and introduced in the pSuper vector. The polIII-dependent transcription unit was then 
introduced in the green fluorescent lentiviral vector $\mathrm{PTRIP} / \Delta \mathrm{U} 3$-EF1 $\alpha$ (TRIP/ $\Delta U 3$-EF1 $\alpha$-GFP), as previously described (56). An shRNA directed against the luciferase protein was used as control. Production of shSTAT3, shSTAT5, and shRNA control lentiviral vectors was performed as described previously (57). IEL lines $\left(5 \times 10^{5} \mathrm{cells} / \mathrm{ml}\right)$ were incubated with the indicated lentiviral particles at MOI of 10 for 24 hours and then intensively washed. Cells were then maintained in culture for a few days before sorting $\mathrm{GFP}^{+}$cells by FACS on a ALTRA (Dakocytomation). Transduction efficiency, expressed as percent $\mathrm{GFP}^{+}$cells, was measured by FACS analysis and reached 20\%-30\%; shRNA efficiency and biological consequences were all investigated on sorted $\mathrm{GFP}^{+}$cells.

Flow cytometry analysis. Lymphocytes $\left(10^{5}\right)$ were incubated with PE-, FITC-, allophycocyanin-Cy-chome- (APC-), or PerCPcy5.5-conjugated (PerCP-conjugated) mAbs against human CD4, CD8, CD3, CD45, and CD103 (BD Biosciences) at optimal concentrations for 20 minutes at $4^{\circ} \mathrm{C}$. For intracellular staining, $5 \times 10^{5}$ cells, fixed in Fix Buffer I at $37^{\circ} \mathrm{C}$ for 10 minutes and permeabilized in Perm Buffer III (BD Biosciences) at $4^{\circ} \mathrm{C}$ for 30 minutes, were labeled with PE-conjugated mAbs to human phospho-STAT5(Y694), phospho-STAT3(Y705), phospho-ERK1/2(pT202/pY204), phospho-Akt(pT308), or control isotype (all reagents from BD Biosciences) at optimal concentrations at $20^{\circ} \mathrm{C}$ for 1 hour. For intracellular detection of Bcl-2 and Bcl-xL, $10^{5}$ cells were fixed and permeabilized using BD Cytoperm/cytofix and labeled with PE-conjugated anti-human Bcl-xL mAb (Abcam), FITC-conjugated anti-human Bcl-2 $\mathrm{mAb}$ (DAKO), or control isotypes at $4{ }^{\circ} \mathrm{C}$ for 20 minutes. For detection of apoptosis, $10^{5}$ cells were stained with annexin V-FITC and PI (TACS kit; R\&D Systems). Labeled cells were analyzed with a BD-LSR II apparatus and CELLQuest software (BD Biosciences).

Mouse in vivo treatment with AMG714 or ABT-737. To test the in vivo effect of AMG714, 2 groups of $5 \mathrm{IL}-15 \mathrm{Tg}_{\mathrm{E}}$ mice (31) received 2 i.p. injections,

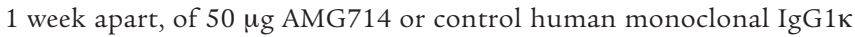
(clone 120.6.1 G1k). To test the effect of ABT-737, 3 IL-15Tg mice and 2 B6 mice were given daily i.p. injections of ABT-737 $(75 \mathrm{mg} / \mathrm{kg})$ diluted in DMSO and a mixture of $30 \%$ propylene glycol, $5 \%$ Tween $80,65 \%$ D5W (5\% dextrose in water), $\mathrm{pH} 4-5$ (final DMSO concentration, $1 \%$; ref. 23 ). The same numbers of mice were sham treated with vehicle only. Mice were euthanized on day 14, and lymphocytes were isolated from lamina propria, intestinal epithelium, and/or mesenteric lymph nodes. Absolute numbers of $\mathrm{CD}^{+} \mathrm{T}$ cells were quantified by flow cytometry using PE-conjugated mouse anti-CD $3 \mathrm{mAb}$ and Trucount beads (BD Biosciences). Blood samples were collected at days 0,7 , and 14 for flow cytometry assessment of percentages and total numbers of $\mathrm{CD} 4^{+}$and $\mathrm{CD} 8^{+} \mathrm{T}$ lymphocytes as well as NK and NKT cells. To study lymphocyte apoptosis, IL-15Tg $\mathrm{S}_{\mathrm{E}}$ mice were sacrificed 2 days after a single i.p. injection of $50 \mu \mathrm{g}$ AMG714. Lymphocytes were isolated and stained with eFluor450-conjugated anti-CD45 and APC-conjugated anti-CD3 mAbs (BD Biosciences) and TACS kit (R\&D Systems). Protocols were approved by Ile-de-France- René Descartes Ethics committee for animal experimentation.

Immunohistochemistry. Immunohistochemical staining of formalin-fixed and paraffin-embedded human duodenal biopsies were performed on 5 - $\mu \mathrm{m}$ sections after antigen retrieval with $10 \mathrm{mM}$ citrate buffer ( $\mathrm{pH} \mathrm{6}$ ). Sections were incubated with rabbit polyclonal $\mathrm{Ab}$ against phosphoJAK3(Y980) (1 $\mu \mathrm{g} / \mathrm{ml}$; Santa Cruz Biotechnology) and mouse anti-CD3 $\mathrm{mAb}$ (DakoCytomation) at $20^{\circ} \mathrm{C}$ for 1 hour, or with rabbit anti-phosphoSTAT5(Y694) mAb (1:100 dilution; Epitomics) at $4^{\circ} \mathrm{C}$ overnight. Ab binding was detected using DAKO REAL detection system and DAB chromogen (DakoCytomation). Sections were counterstained with modified Harris hematoxylin solution (Sigma-Aldrich). Immunohistochemical labeling of $7-\mu \mathrm{m}$-thick acetone-fixed cryostat sections of frozen mouse duodenum was performed by incubation with rabbit anti-KI67 $\mathrm{Ab}(3 \mu \mathrm{g} / \mathrm{ml}$; Abcam) and hamster anti-CD $3 \varepsilon \mathrm{mAb}(1 \mu \mathrm{g} / \mathrm{ml}$; BD Biosciences $)$ at $20^{\circ} \mathrm{C}$ for 1.5 hours followed by FITC-conjugated goat anti-rabbit $\mathrm{Ab}(20 \mu \mathrm{g} / \mathrm{ml}$; Abcam $)$ and Cy3-conjugated anti-hamster $\mathrm{Ab}(13 \mu \mathrm{g} / \mathrm{ml}$; Jackson ImmunoResearch Laboratories) at $20^{\circ} \mathrm{C}$ for 1 hour. Slides were mounted in Vectashield medium containing DAPI (Vector Laboratories) and examined with an epifluorescence microscope (Zeiss) equipped with a CCD camera (Photometrics).

Real-time PCR. Total RNA from IEL lines was extracted with RNeasy Mini Kit (Qiagen) and reverse transcribed as described previously (6). Bcl-2, Bcl-xL, and Mcl-1 mRNAs were quantified by real-time PCR using TaqMan universal PCR Master Mix, corresponding primers (Applied Biosystems), and 40 cycles of denaturation $\left(95^{\circ} \mathrm{C}, 15\right.$ seconds) and annealing/extension $\left(60^{\circ} \mathrm{C}\right.$, 1 minute) on an Applied Biosystems 7300 Real-Time PCR apparatus. Data were normalized referring to ribosomal Protein Large PO.

Western blot. Cell pellets or duodenal biopsies were lysed in RIPA buffer (50 mM Tris-HCl, pH 7.4; 1\% NP-40; $0.25 \%$ sodium deoxycholate; $150 \mathrm{mM}$ $\mathrm{NaCl}$; and $1 \mathrm{mM}$ EDTA) supplemented with $0.5 \mathrm{mM}$ PMSF, phosphatase inhibitor cocktails I and II (Sigma-Aldrich), and protease inhibitor cocktail (Roche Diagnosis) at $4^{\circ} \mathrm{C}$ for 1 hour.

Proteins were separated in 10\%-12\% SDS-PAGE gels under reducing conditions, transferred to PVDF membranes (Bio-Rad Laboratories), and probed with mAbs against Bcl-xL, Mcl-1, Akt1, actin (Santa Cruz Biotechnology), or phospho-STAT3(Y705) (Cell signaling Technology) or with polyclonal rabbit Ab against Bcl-2 (Biolegend), phospho-STAT5(Y694), STAT5, STAT3, cleaved caspase-3(D175), histone H3 (Cell Signaling Technology), or anti-phospho-Akt1/2/3 (Santa Cruz Biotechnology). Primary Abs were revealed by HRP-conjugated donkey anti-rabbit IgG (Cell Signaling Technology) or sheep anti-mouse IgG (GE Healthcare). To control for loading differences, blots were stripped and reprobed with Abs against actin, histone $\mathrm{H} 3$, or the corresponding unphosphorylated signaling molecules. Binding of secondary Abs was visualized using chemiluminescence (ECL kit; GE Healthcare).

To analyze Jak3 phosphorylation, $200 \mu \mathrm{g}$ of protein extracts in RIPA buffer were incubated with $1 \mu \mathrm{g}$ rabbit anti-Jak3 polyclonal $\mathrm{Ab}$ (Abcam) and $20 \mu \mathrm{l}$ protein $\mathrm{G}$ agarose beads (GE Healthcare) at $4^{\circ} \mathrm{C}$ for 1 hour. Immunoprecipitates were washed, boiled, separated on a $10 \%$ SDS-PAGE gel, and transferred to PVDF membrane. Membranes were blotted with anti-phosphotyrosine mAb 4G10 (1:1,000; Upstate Millipore) and then with anti-Jak3 $\mathrm{Ab}(2 \mu \mathrm{g} / \mathrm{ml}$; Abcam).

Statistics. Paired 2-tailed $t$ test and 1-way ANOVA with Tukey's multiple comparison test were used to compare in vitro efficiency of different inhibitors. Results in groups of patients and mice were compared using MannWhitney test. A $P$ value less than 0.05 was considered significant.

\section{Acknowledgments}

This work was supported by INSERM, ARC, Foundation Princesse Grace de Monaco, and AFDIAG. G. Malamut was supported by ARC; R. El Machhour was supported by la Région Ile-de-France. The authors are grateful to Y. Jacques, Abbott Laboratories, and AMGEN Company for providing reagents; P. Wind and A. Berger for providing surgical duodenal samples; and N. Guegan and G. Pivert for technical support.

Received for publication October 5, 2009, and accepted in revised form February 24, 2010.

Address correspondence to: Nadine Cerf-Bensussan or Bertrand Meresse, INSERM U989, Université René Descartes, Faculté de Médecine 5, 156 rue de Vaugirard, 75730 Paris Cedex 15, France. Phone: 33.140615302; Fax: 33.140615638; E-mail: nadine.cerfbensussan@inserm.fr (N. Cerf-Bensussan); bertrand.meresse@ inserm.fr (B. Meresse). 
1. Meresse B, Ripoche J, Heyman M, Cerf-Bensussan $\mathrm{N}$. Celiac disease: from oral tolerance to intestinal inflammation, autoimmunity and lymphomagenesis. Mucosal Immunol. 2009;2(1):8-23.

2. Daum S, et al. Intestinal non-Hodgkin's lymphoma: a multicenter prospective clinical study from the German study group on intestinal non-hodgkin's lymphoma. J Clin Oncol. 2003;21(14):2740-2746.

3. Cellier C, et al. Refractory sprue, coeliac disease, and enteropathy-associated T-cell lymphoma. French coeliac disease study group. Lancet. 2000;356(9225):203-208.

4. Spencer J, et al. Changes in intraepithelial lymphocyte subpopulations in coeliac disease and enteropathy associated $\mathrm{T}$ cell lymphoma (malignant histiocytosis of the intestine). Gut. 1989;30(3):339-346.

5 . Hue $S$, et al. A direct role for NKG2D/MICA interaction in villous atrophy during celiac disease. Immunity. 2004;21(3):367-377.

6. Mention JJ, et al. Interleukin 15: a key to disrupted intraepithelial lymphocyte homeostasis and lymphomagenesis in celiac disease. Gastroenterology. 2003;125(3):730-745.

7. Meresse B, et al. Coordinated induction by IL15 of a TCR-independent NKG2D signaling pathway converts CTL into lymphokine-activated killer cells in celiac disease. Immunity. 2004;21(3):357-366.

8. Meresse B, et al. Reprogramming of CTLs into natural killer-like cells in celiac disease. J Exp Med. 2006;203(5):1343-1355.

9. Fehniger TA, et al. Fatal leukemia in interleukin 15 transgenic mice follows early expansions in natural killer and memory phenotype CD8+ T cells. J Exp Med. 2001;193(2):219-231.

10. Di Sabatino A, et al. Epithelium derived interleukin 15 regulates intraepithelial lymphocyte Th1 cytokine production, cytotoxicity, and survival in coeliac disease. Gut. 2006;55(4):469-477.

11. Maiuri L, et al. Interleukin 15 mediates epithelial changes in celiac disease. Gastroenterology. 2000;119(4):996-1006

12. Tjon JM, et al. Defective synthesis or association of T-cell receptor chains underlies loss of surface T-cell receptor-CD3 expression in enteropathy-associated T-cell lymphoma. Blood. 2008;112(13):5103-5110.

13. Cellier $\mathrm{C}$, et al. Abnormal intestinal intraepithelial lymphocytes in refractory sprue. Gastroenterology. 1998;114(3):471-481.

14. Verkarre V, et al. Recurrent partial trisomy 1q22-q44 in clonal intraepithelial lymphocytes in refractory celiac sprue. Gastroenterology. 2003;125(1):40-46.

15. Malamut G, et al. Presentation and long-term follow-up of refractory celiac disease: comparison of type I with type II. Gastroenterology. 2009;136(1):81-90.

16. Budagian V, Bulanova E, Paus R, Bulfone-Paus S. IL-15/IL-15 receptor biology: a guided tour through an expanding universe. Cytokine Growth Factor Rev. 2006;17(4):259-280.

17. Fehniger TA, Caligiuri MA. Interleukin 15: biology and relevance to human disease. Blood. 2001;97(1):14-32.

18. Huntington ND, et al. Interleukin 15-mediated survival of natural killer cells is determined by interactions among Bim, Noxa and Mcl-1. Nat Immunol. 2007;8(8):856-863.

19. Carson WE, et al. A potential role for interleukin15 in the regulation of human natural killer cell survival. J Clin Invest. 1997;99(5):937-943.

20. Zheng X, et al. Bcl-xL is associated with the anti-apoptotic effect of IL-15 on the survival of CD56(dim) natural killer cells. Mol Immunol. 2008;45(9):2559-2569.

21. Oh S, et al. IL-15 as a mediator of CD4+ help for CD8 + T cell longevity and avoidance of TRAILmediated apoptosis. Proc Natl Acad Sci US A. 2008;105(13):5201-5206.

22. Chu CL, Chen SS, Wu TS, Kuo SC, Liao NS. Differential effects of IL-2 and IL-15 on the death and survival of activated TCR gamma delta+ intestinal intraepithelial lymphocytes. J Immunol. 1999;162(4):1896-1903

23. Cragg MS, Harris C, Strasser A, Scott CL. Unleashing the power of inhibitors of oncogenic kinases through BH3 mimetics. Nat Rev Cancer. 2009;9(5):321-326

24. Oltersdorf T, et al. An inhibitor of Bcl-2 family proteins induces regression of solid tumours. Nature. 2005;435(7042):677-681.

25. Mortier E, et al. Soluble interleukin-15 receptor alpha (IL-15R alpha)-sushi as a selective and potent agonist of IL-15 action through IL-15R beta/ gamma. Hyperagonist IL-15 $\times$ IL-15R alpha fusion proteins. J Biol Chem. 2006;281(3):1612-1619.

26. Huntington ND, et al. IL-15 trans-presentation promotes human NK cell development and differentiation in vivo. J Exp Med. 2009;206(1):25-34.

27. Schluns KS, et al. Distinct cell types control lymphoid subset development by means of IL-15 and IL-15 receptor alpha expression. Proc Natl Acad Sci US A. 2004;101(15):5616-5621.

28. Ellery JM, Nicholls PJ. Alternate signalling pathways from the interleukin-2 receptor. Cytokine Growth Factor Rev. 2002;13(1):27-40.

29. Changelian PS, et al. The specificity of JAK3 kinase inhibitors. Blood. 2008;111(4):2155-2157.

30. Baslund B, et al. Targeting interleukin-15 in patients with rheumatoid arthritis: a proof-of-concept study. Arthritis Rheum. 2005;52(9):2686-2692.

31. Ohta N, et al. IL-15-dependent activationinduced cell death-resistant Th1 type CD8 alpha beta+NK1.1 $+\mathrm{T}$ cells for the development of small intestinal inflammation. J Immunol. 2002;169(1):460-468

32. Lodolce JP, et al. IL-15 receptor maintains lymphoid homeostasis by supporting lymphocyte homing and proliferation. Immunity. 1998;9(5):669-676.

33. Suzuki H, Duncan GS, Takimoto H, Mak TW. Abnormal development of intestinal intraepithelial lymphocytes and peripheral natural killer cells in mice lacking the IL-2 receptor beta chain. J Exp Med. 1997;185(3):499-505.

34. Bernardo D, et al. Higher constitutive IL15R alpha expression and lower IL-15 response threshold in coeliac disease patients. Clin Exp Immunol. 2008;154(1):64-73.

35 . Hodge DL, et al. Interleukin-15 enhances proteasomal degradation of bid in normal lymphocytes: implications for large granular lymphocyte leukemias. Cancer Res. 2009;69(9):3986-3994.

36. Ghoreschi K, Laurence A, O'Shea JJ. Janus kinases in immune cell signaling. Immunol Rev. 2009;228(1):273-287.

37. KellyJ,etal.Arole for Stat 5 in CD8+ T cell homeostasis JImmunol. 2003;170(1):210-217.

38. Yao Z, et al. Stat5a/b are essential for normal lymphoid development and differentiation. Proc Natl Acad Sci U S A. 2006;103(4):1000-1005.

39. Buettner R, Mora LB, Jove R. Activated STAT signaling in human tumors provides novel molecular targets for therapeutic intervention. Clin Cancer Res. 2002;8(4):945-954
40. Turkson J, Jove R. STAT proteins: novel molecular targets for cancer drug discovery. Oncogene. 2000;19(56):6613-6626.

41. Grillot DA, et al. Genomic organization, promoter region analysis, and chromosome localization of the mouse bcl-x gene. J Immunol. 1997; 158(10):4750-4757.

42. Nelson EA, Walker SR, Alvarez JV, Frank DA. Isolation of unique STAT5 targets by chromatin immunoprecipitation-based gene identification. J Biol Chem. 2004;279(52):54724-54730.

43. Epling-Burnette PK, et al. Inhibition of STAT3 signaling leads to apoptosis of leukemic large granular lymphocytes and decreased Mcl-1 expression. J Clin Invest. 2001;107(3):351-362.

44. Yamasaki S, et al. Growth and apoptosis of human natural killer cell neoplasms: role of interleukin2/15 signaling. Leuk Res. 2004;28(10):1023-1031.

45. Berard M, Brandt K, Bulfone-Paus S, Tough DF. IL-15 promotes the survival of naive and memory phenotype CD8+ T cells. J Immunol. 2003;170(10):5018-5026.

46. Ilangumaran S, et al. Suppressor of cytokine signaling 1 attenuates IL- 15 receptor signaling in CD8+ thymocytes. Blood. 2003;102(12):4115-4122.

47. Yajima $\mathrm{T}$, et al. IL-15 regulates CD8+ $\mathrm{T}$ cell contraction during primary infection. $J$ Immunol. 2006;176(1):507-515.

48. Nakazato K, et al. Enforced expression of Bcl-2 partially restores cell numbers but not functions of TCRgammadelta intestinal intraepithelial $\mathrm{T}$ lymphocytes in IL-15-deficient mice. J Immunol. 2007;178(2):757-764.

49. Mueller DL, Seiffert S, Fang W, Behrens TW. Differential regulation of bcl-2 and bcl-x by CD3, CD28, and the IL-2 receptor in cloned CD4+ helper T cells. A model for the long-term survival of memory cells. J Immunol. 1996;156(5):1764-1771.

50. Zaunders JJ, et al. Polyclonal proliferation and apoptosis of CCR5+ T lymphocytes during primary human immunodeficiency virus type 1 infection: regulation by interleukin (IL)-2, IL-15, and Bcl-2. J Infect Dis. 2003;187(11):1735-1747.

51. Hildeman DA, Mitchell T, Kappler J, Marrack P. $\mathrm{T}$ cell apoptosis and reactive oxygen species. J Clin Invest. 2003;111(5):575-581.

52. McInnes IB, Leung BP, Sturrock RD, Field M, Liew FY. Interleukin-15 mediates T cell-dependent regulation of tumor necrosis factor-alpha production in rheumatoid arthritis. Nat Med. 1997;3(2):189-195.

53. Ruckert R, et al. Inhibition of keratinocyte apoptosis by IL-15: a new parameter in the pathogenesis of psoriasis? J Immunol. 2000;165(4):2240-2250.

54. Yokoyama S, et al. Antibody-mediated blockade of IL-15 reverses the autoimmune intestinal damage in transgenic mice that overexpress IL-15 in enterocytes. Proc Natl Acad Sci U S A. 2009;106(37):15849-15854.

55. Cerf-Bensussan N, Guy-Grand D, Griscelli C. Intraepithelial lymphocytes of human gut: isolation, characterisation and study of natural killer activity. Gut. 1985;26(1):81-88.

56. Brunet de la Grange P, et al. Low SCL/TAL1 expression reveals its major role in adult hematopoietic myeloid progenitors and stem cells. Blood. 2006;108(9):2998-3004.

57. Sirven A, et al. Enhanced transgene expression in cord blood CD34(+)-derived hematopoietic cells, including developing $\mathrm{T}$ cells and NOD/SCID mouse repopulating cells, following transduction with modified trip lentiviral vectors. Mol Ther. 2001;3(4):438-448. 